\title{
Light-induced heat-conducting micro/nano spheroidal particles and their thermoosmotic velocity fields.
}

\author{
EJ Avital ${ }^{1}$ and T Miloh ${ }^{2}$ \\ ${ }^{1}$ School of Engineering and Materials Science, Queen Mary University of London, London E1 4NS \\ UK, email: e.avital@qmul.ac.uk \\ ${ }^{2}$ School of Mechanical Engineering, Tel Aviv University, Tel-Aviv 69978, Israel, email: \\ miloh@eng.tau.ac.il
}

\begin{abstract}
A micro/nano spheroidal conducting particle embedded in a fluid of a uniform ambient temperature is considered for its temperature and the induced velocity fields due to thermoosmosis. The particle is assumed to be uniformly heated using for example continuous light irradiation by a conventional laser. This is a model problem of thermoplasmonics, where nano or micro particles are used for heat storage and release as in medical therapy and imaging purposes. The temperature field is governed by the Poisson heat diffusion equation and the self-induced thermoosmotic flow (STOF) field is taken as a Stokes type. Analytical closed solutions are derived for both the temperature distributions (inside and outside the particle) as well as for the STOF in the solute for both prolate and oblate configurations. They are based on using spheroidal harmonics expressed in terms of Legendre functions, where owing to orthogonality only a few terms are needed to fully prescribe the entire field. The analytical solutions thus obtained are also numerically verified for few selected cases.

Results for conducting spheroidal particles with inner thermal conductivities lower or higher than the outer medium's conductivity are analysed. It is shown that the surface temperature of the particle is always highest at the tip nearest to the spheroid's centre. However, the location of the peak of the surface heat flux depends on the precise ratio between the inner and outer thermal conductivities, where it peaks at the nearest tip for a low inner thermal conductivity and at the farthest tip for a high inner thermal conductivity. Plots of temperature and heat flux distributions over the surface of both prolate and oblate spheroidal particles as well as the thermoosmotic (Soret) induced velocity, vorticity and stream-function in the liquid phase are given and analysed.
\end{abstract}

Keywords: Heat equation, Stokes flow, light-induced Joule heating, micro particle, thermoplasmonics 


\section{List of symbols:}

$A_{n} \quad$ - Coefficient in the Legendre series solution for $T_{\text {in }}$ in the prolate spheroidal particle

$B_{n} \quad-$ Coefficient in the Legendre series solution for $T_{\text {out }}$ outside the prolate spheroidal particle

$A_{n}^{*} \quad$ - Coefficient in the Legendre series solution for $T_{\text {in }}$ in the oblate spheroidal particle

$\mathrm{B}^{*}{ }_{\mathrm{n}} \quad$ - Coefficient in the Legendre series solution for $\mathrm{T}_{\text {out }}$ outside the oblate spheroidal particle

c - Parameter in the spheroidal coordinates

$\mathrm{D}_{\mathrm{T}} \quad$ - Thermal (Soret) transport parameter

D - Spheroid's length

d - Spheroid's diameter

$G_{k} \quad$ - Coefficient of the function $I_{k}$ in the induced velocity solution

h - Scale factor of the spheroidal coordinates

$\mathrm{I}_{\mathrm{k}} \quad$ - Elliptic integral function in the induced velocity solution

k - Thermal conductivity

n $\quad$ - Normal to the particle's surface

$\mathrm{P}_{\mathrm{n}} \quad$ - Legendre function of the first kind

$\widetilde{Q}_{0} \quad$ - Overall heat source in the particle

$\mathrm{Q}_{\mathrm{n}} \quad$ - Legendre function of the second kind

$\mathrm{q}_{0} \quad$ - Heat density inside the particle

$\mathrm{R} \quad$ - Sphere's radius

r $\quad$ - Radial distance

$\mathrm{T}$ - Temperature

u - Velocity

$\mathrm{x} \quad$ - The spheroid's major axis containing the length $\mathrm{D}$

$\mathrm{y}$ and $\mathrm{z}$ - The spheroid's minor axes containing the diameter $d$

$\mu \quad$ - Spheroidal coordinate that is parallel to the spheroid's surface

$\xi \quad$ - Prolate spheroidal coordinate that is normal to the spheroid's surface

$\zeta$ - Oblate spheroidal coordinate that is normal to the spheroid's surface

$\Phi \quad$ - Velocity potential

$\phi \quad$ - Polar angle

$\omega \quad$ - Vorticity

$\psi \quad$ - Stream-function

Subscripts:

ave - Particle's surface-averaged property

equ - Property of the equivalent sphere, i.e. having the same volume as the spheroid

in - Property that is inside the particle

out - Property that is outside the particle 


\section{Introduction}

The temperature fields within and over the surface of nano and micro particles has received considerable interest since the Soret effect was discovered in the nineteen century, when it was found that an imposed ambient temperature gradient can be used to transport small heat-conducting particles [1, 2]. This phenomenon, which is also related to thermal diffusion or thermophoresis, has a range of applications from aerosols to microgravity manufacturing [2]. The transport of small particles under the influence of an ambient temperature gradient can be modelled using the linearized forced heat diffusion equation and the assumption of Stokes flow in the liquid phase due to the very low Reynolds number, while the continuum assumption still holds [2].

Modelling thermophoresis usually concentrated on the case of an isolated conducting particle while assuming an imposed uniform ambient temperature gradient and no heat sources both inside or outside the particle. Closed analytical solutions were found for the perfectly symmetric case of a spherical particle [2]. The configuration of a spheroidal particle was also investigated where analytical solution for the temperature field was sought using a series expansion based on the Legendre functions [3]. A similar approach can also be used to solve for the self-induced thermoosmotic flow (STOF) velocity field in the solute around the particle due to light irradiation (internal Joule heating). This results in a non-uniform pressure distribution over the particle's surface, or a Soret-type surface slip velocity, which may lead to a finite mobility under some symmetry-breaking effects. Numerical solutions for non-spherical configurations, can also be based on employing a series expansion of the Legendre functions or other common numerical approaches as finite difference and finite element. For further details on thermophoresis studies using analytical, experimental and computational means, the reader is referred to Refs [1] and [2].

An interesting approach of achieving thermophoresis of spheroidal micro/nano particles without imposing external temperature gradient, i.e. having a uniform temperature very far from the particle, was proposed by Miloh \& Nagler [4]. Instead of having the non-uniformity in the pressure caused by the ambient conditions, it is caused by non-uniformity of the two-face Janus particle. The particle was proposed to be built by two halves (Janus) having different thermal conductivities, which results in a material symmetry breaking. The particle can be heated using for example light irradiation by a common (e.g. Helium-Neon) laser. This will cause a heat source (Joule heating) inside the particle, a non-uniform temperature distribution over the particle's surface, a Soret-type surface slip velocity, an induced flow field around the particle and a non-uniform pressure field that can propel the particle. Spheroidal particles were considered, where again the Poisson heat equation was simply used as the governing equation for the internal temperature field. The external STOF 
field was taken to be of a Stokes type (ignoring inertia versus viscous effects). Analytical solutions based on Legendre functions were then derived to investigate the particle's mobility resulting in simplified expressions for its mobility velocity.

Experimental observation of the self thermophrosis of a spherical Janus micro particle was reported by Jiang et al [5]. Half-metal particles were exposed to laser irradiation causing a local temperature gradient, yielding a non-symmetric thermal slip velocity and turning the particles to colloidal swimmers. Modelling based on spherical harmonics was also suggested. Further experimental evidence of the self propulsion of Janus particles was given by Moyses et al [6], who looked at the motion near a flat glass, revealing sinusoidal rosette-type patterns. A theoretical model was derived by treating the particle as point of singularly. Wei and Jan [7] proposed a generalised model for the motion of a pair of particles based on a multipole expansion for the Stokes flow field. Several time scales were discovered and the stability of the particle's motion was discussed.

The studies noted above brings us to the case studied in this paper, where a conducting homogeneous (symmetric) spheroidal particle is uniformly heated by a laser embedded in a fluid having a uniform ambient temperature. The particle can be of a prolate spheroidal geometry i.e. $\mathrm{D}>\mathrm{d}$ as in Fig 1 illustrating the problem schematics, or it can be of an oblate spheroidal geometry, i.e. $\mathrm{D}<\mathrm{d}$. . This is typically the case of using particles for heat generation and storage as in thermoplasmonics of metal nano and micro particles [8] of arbitrary shape. However, it is important to note that there is a major difference between the case of spherical or spheroidal particles. It is well known that for a perfectly symmetric spherical shape the self-induced surface temperature gradient is uniform and thus there is no thermophoresis! Nevertheless, interestingly , the self-induced surface temperature for spheroidal particles is not uniform and thus there is a finite temperature gradient on the surface which results in a finite Soret slip that drives a non-zero STOF in the liquid phase. Such a phenomenon is of interest due to potential applications in several fields ranging from medical cancer therapy, drug and gene delivery, heat-assisted magnetic recording to photoacoustic and photo-thermal imaging [8].

A review on heat generation using illuminated nano particles was given by Govorov and Richardson [9]. A model based on a spherical particle was presented and the heat generation was perceived to be affected by the accumulative effect of the particles and by the Coulomb interaction. An experimental and theoretical study was reported by Richardson et al [10]. It showed that the efficiency of light- to- heat conversion was close to one, resulting in a temperature rise by a few Celsius using a small common laser illuminating a large concentration of particles. A more recent 
review was provided by Pustovalov [11] for the heat generation by laser irradiated metal particles. An intesting model was suggested for an assembly of particles, starting from a single spherical particle then accounting for other particles while making clear separation between the various time scales for the transient problem. The use of heat-releasing nano particles for medical treatment was reviewed by Jaque et al [12]. Finally, it is worth mentioning that plasmonic heating of nanoparticles and metamaterials, is not necessarily restricted to laser illumination and can be also attained by sunlight, in connection for example with solar energy harvesting and solar vapour generation [13, $14]$.

At the heart of the modelling of the problem, it is assumed as in the case of thermophoresis, that the heat equation governs the temperature field and the flow field induced due by the temperature field is of Stokes type $[8,13]$. Hence the equations for the temperature fields inside and outside the particle are simply taken under the assumption that heat diffusion is the governing effect and heat convection can be ignored due to small thermoosmotic velocities, as [8];;

$$
\begin{gathered}
k_{\text {in }} \nabla^{2} T_{\text {in }}=-q_{0}, \\
\nabla^{2} T_{\text {out }}=0 .
\end{gathered}
$$

Eq (1) holds inside the particle and Eq (2) holds in the medium outside of the particle. Commonly, the thermal conductivities $\mathrm{k}_{\text {in }}$ and $\mathrm{k}_{\text {out }}$, as well as the heat density source $\mathrm{q}_{0}$ are taken as uniform [8, 13]. This is an acceptable approximation due to the small variation of the temperature and the small size of the particle and in accordance with the Rayleigh quasi-static assumption.

The temperature fields $T_{\text {in }}$ and $T_{\text {out }}$ are matched at the particle's surface by requiring continuity in both the temperature and the heat flux normal to the particle's surface [8];

$$
\begin{gathered}
T_{\text {in }}=T_{\text {out }}, \\
k_{\text {in }} \frac{\partial T_{\text {in }}}{\partial n}=k_{\text {out }} \frac{\partial T_{\text {out }}}{\partial n},
\end{gathered}
$$

where $n$ is the normal to the particle's surface. A simple analytical solution of Eqs (1-4), can then be derived for a spherical particle with a radius $R[8]$;

$$
\begin{gathered}
T_{\text {in }}=\frac{\widetilde{Q}_{0}}{8 \pi k_{\text {in }} R}\left[1+2 \frac{k_{\text {in }}}{k_{\text {out }}}-\left(\frac{r}{R}\right)^{2}\right], r \leqslant R, \\
T_{\text {out }}=\frac{\widetilde{Q}_{0}}{4 \pi k_{\text {out }}} \frac{R}{r}, r \geqslant R,
\end{gathered}
$$

where $\widetilde{Q}_{0}$ denotes the total heat source inside the particle i.e. $\widetilde{Q}_{0}=4 q_{0} \pi R^{3} / 3$ and $\mathrm{r}$ is the radial distance from the particle's centre. The ambient temperature was taken as zero, i.e. 
$T_{\text {out }}(r \rightarrow \infty)=0$, without losing any generality in the solution because of the linear nature of the problem. Interestingly, $\mathrm{T}_{\text {out }}$ does not depend on the particle's thermal conductivity $\mathrm{k}_{\text {in }}$ and this also means that the temperature on the surface of the particle does not depend on $k_{\text {in }}$ due to the continuity of the temperature on the particle's surface. There is no induced flow field outside of the particle because of the uniformity of the temperature on the particle's surface. Since the surface temperature over the sphere is constant there is no surface gradient and hence no slip-velocity to drive STOF in the solute about a spherical particle.

The situation for non-spherical shapes is somewhat different. A general analytical solution for a triaxial ellipsoidal particle embedded in a medium with a uniform ambient temperature was recently derived by Miloh [15] using Làme functions. The non-spherical geometry was shown to cause a temperature variation along the surface of the particle and hence a thermoosmotic flow field was induced around it. An analytical solution for the induced velocity field was derived using a decomposition of ellipsoidal harmonics and the use of elliptic integral functions. The limiting case of a spheroid was considered for its surface-averaged temperature.

The thermoosmotic Soret slip velocity over the particle's surface is commonly taken as linearly relative to the temperature gradient along the surface, i.e. $\vec{u}_{\text {slip }}=-D_{T} \nabla_{s} T$, where $\mathrm{D}_{\mathrm{T}}$ is the thermopheretic mobility. Brenner [16] suggested for example the following expression $D_{T}=\gamma\left(1+2 k_{\text {in }} / k_{\text {out }}\right) \quad$ for non-inert particles, where $\gamma$ is the product of the diffusivity and the thermal expansion of the surrounding fluid. Yet, another simple explicit expression for the thermal mobility term for the case of a charged particle immersed in an electrolyte, is provided by Wurger [17], in terms of the particle's surface zeta potential. The above linear slip-velocity model, is generally valid for uncharged or weakly charged nano/micro particles such that the interaction extent between the colloid and surrounding liquid, is on a length scale small compared to the characteristic size of the particle. In the context of thermophoresis or termoelectric (Seebeck) effect in the presence of an electric double layer, this is equivalent to the assumption of a small Debye scale [17], which is also supported by various molecular simulations [18].

In this study we seek to extend and simplify the above analytical solution for both prolate and oblate spheroidal configurations. Solutions based on the more familiar Legendre functions will be derived to show a closed form of analytic solution and will also be numerically verified. The ratio of the thermal conductivities $\mathrm{k}_{\mathrm{in}} / \mathrm{k}_{\text {out }}$ between the two phases, can considerably vary where typically the surrounding medium is of water, yielding $\mathrm{k}_{\text {out }}=0.6(\mathrm{~W} / \mathrm{m} \mathrm{K})$. The spheroid's thermal conductivity 
$\mathrm{k}_{\text {in }}$ can vary from $0.04 \mathrm{~W} /(\mathrm{m} \mathrm{K})$ for polystyrene to $8 \mathrm{~W} /(\mathrm{m} \mathrm{K})$ for Beryllium copper, $35 \mathrm{~W} /(\mathrm{m} \mathrm{K})$ for lead and even $400 \mathrm{~W} /(\mathrm{m} \mathrm{K})$ for gold. In this case we will concentrate on e particles of polystyrene and Beryllium copper, where higher values of $k_{i n}$ as of lead resulted in a physical behaviour similar to that of the Beryllium copper case. The solution methodology is derived in the next section, followed by results and analysis and concluded by a summary section.

\section{Solution methodology}

As noted in the introduction, the problem consists of a prolate or an oblate spheroid embedded in a medium with a constant uniform ambient temperature. For simplicity we will take that ambient temperature as zero without losing the generality of the problem due to the linearity of the governing equations (1) to (4). The spheroid has a thermal conductivity $\mathrm{k}_{\text {in }}$ that is different than that of the surrounding medium's $\mathrm{k}_{\text {out }}$. Uniform heat density $\mathrm{q}_{0}$ is assumed inside the spheroid as expressed in Eq (1) and no heat source is applied for the surrounding medium as expressed in Eq (2). It results in a Poisson equation for $T_{\text {in }}$ the temperature inside the spheroid and in a Laplace equation for $\mathrm{T}_{\text {out }}$ the temperature outside the spheroid. The induced velocity field is assumed to follow Stokes flow due to the very low Reynolds number, while the continuum assumption still holds.

Firstly, the analytical solution for the temperature fields is presented. Its derivation is similar to that of Key \& Chang [3] and Miloh \& Nagler [4], but a simplification is achieved due the assumption of a uniform ambient temperature. Secondly, the analytical solution of the induced termoosmotic velocity field is presented. Its derivation relies on the approach of Miloh [15] for a general triaxial ellipsoid, where again a significant simplification is achieved because of the axisymmetry of the spheroid case. Finally, the numerical approach used for the verification of the analytical solutions is presented.

\subsection{The temperature field solution}

The prolate spheroid case can be dealt with using the following spheroidal coordinates $(\xi, \mu, \phi)$ [7];

$$
x=c \mu \xi, \quad y+i z=c \sqrt{\left(1-\mu^{2}\right)\left(\xi^{2}-1\right)} e^{i \phi},
$$

where $\mathrm{x}$ is the Cartesian direction of the major axis containing the spheroid's length $\mathrm{D},(\mathrm{y}, \mathrm{z})$ are Cartesian directions of the minor axes containing the spheroid's diameter $\mathrm{d}$ and $i^{2}=-1$. The spheroidal coordinates are bounded as; $\xi \geqslant 1,-1 \leqslant \mu \leqslant 1,0 \leqslant \phi<2 \pi$. The spheroidal surface is 
at $\xi_{0}=1 / \sqrt{1-d^{2} / D^{2}}$, where $c=D / \xi_{0}$ denotes half the distance between the two foci and $\mathrm{D}>\mathrm{d}$. The potential solution of Eqs (1) and (2) can then be written using a Legendre series [4];

$$
\begin{gathered}
T_{\text {in }}(\xi, \mu)=-\frac{q_{0} c^{2}}{6}\left(\xi^{2}+\mu^{2}\right)+\frac{q_{0} c^{2}}{6} \sum_{n=0} A_{n} P_{n}(\xi) P_{n}(\mu), 1 \leqslant \xi \leqslant \xi_{0}, \\
T_{\text {out }}(\xi, \mu)=\frac{q_{0} c^{2}}{6} \sum_{n=0} B_{n} P_{n}(\mu) Q_{n}(\xi), \xi_{0} \leqslant \xi,
\end{gathered}
$$

where $A_{n}$ and $B_{n}$ are coefficients to be determined.

Using the orthogonality properties of the Legendre polynomial $P_{n}(\mu)$ and the identity $\mu^{2}=2 \mathrm{P}_{2}(\mu) / 3+1 / 3[19,20]$, it can be shown by virtue of the explicit (first) term on the right hand side of Eq (8), that the only prevailing (non-vanishing) terms in (8) and (9) correspond to $\left(\mathrm{A}_{0}, \mathrm{~A}_{2}\right)$ and $\left(\mathrm{B}_{0}, \mathrm{~B}_{2}\right)$. Using the boundary conditions of Eqs (3) and (4) that match the temperature and the heat flux at the spheroid's surface, one gets;

$$
\begin{gathered}
A_{0}=\left(\xi_{0}^{2}+\frac{1}{3}\right)-\frac{2 \xi_{0} Q_{0}\left(\xi_{0}\right)}{\dot{Q}_{0}\left(\xi_{0}\right)} \frac{k_{\text {in }}}{k_{\text {out }}}, B_{0}=-\frac{2 \xi_{0}}{\dot{Q}_{0}\left(\xi_{0}\right)} \frac{k_{\text {in }}}{k_{\text {out }}}, \\
A_{2}=\frac{2 / 3}{P_{2}\left(\xi_{0}\right)-\frac{\dot{P}_{2}\left(\xi_{0}\right) Q_{2}\left(\xi_{0}\right)}{\dot{Q}_{2}\left(\xi_{0}\right)} \frac{k_{\text {in }}}{k_{\text {out }}}}, B_{2}=\frac{-2 / 3}{Q_{2}\left(\xi_{0}\right)-\frac{\dot{Q}_{2}\left(\xi_{0}\right) P_{2}\left(\xi_{0}\right)}{\dot{P}_{2}\left(\xi_{0}\right)} \frac{k_{\text {in }}}{k_{\text {out }}}},
\end{gathered}
$$

where the definitions of the Legendre functions $P_{n}$ and $Q_{n}$ can be found for example in Lamb [19] and Chatjigeorhiou [20]. The dot over the $\mathrm{Q}_{2}, \mathrm{I}_{1}$ and $\mathrm{I}_{2}$ means differentiation with respect to $\xi$.

The oblate spheroid case can be similarly dealt using the following coordinates $(\zeta, \mu, \phi)$ [7];

$$
x=c \mu \zeta, y+i z=c \sqrt{\left(1-\mu^{2}\right)\left(\zeta^{2}+1\right)} e^{i \phi},
$$

where $\quad \xi \geqslant 0,-1 \leqslant \mu \leqslant 1,0 \leqslant \phi<2 \pi, \quad \xi_{0}=1 / \sqrt{d^{2} / D^{2}-1}, c=D / \xi_{0} \quad$ and $\mathrm{D}<\mathrm{d}$. The temperature field can be written as;

$$
\begin{gathered}
T_{\text {in }}(\xi, \mu)=-\frac{q_{0} c^{2}}{6}\left(\xi^{2}-\mu^{2}\right)-\frac{q_{0} c^{2}}{6} \sum_{n=0} A_{n}^{*} P_{n}(i \xi) P_{n}(\mu), 0 \leqslant \xi \leqslant \zeta_{0}, \\
T_{\text {in }}(\xi, \mu)=\frac{-q_{0} c^{2}}{6} \sum_{n=0} B_{n}^{*} P_{n}(\mu) Q_{n}(i \xi), \zeta_{0} \leqslant \zeta .
\end{gathered}
$$

Again only the terms that correspond to $n=0$ and 2 exist. Matching the inner and outer temperature fields using Eqs (3) and (4) leads to;

$$
A_{0}^{*}=\left(\frac{1}{3}-\zeta_{0}^{2}\right)+\frac{2 \zeta_{0} Q_{0}\left(i \zeta_{0}\right)}{\dot{Q}_{0}\left(\zeta_{0}\right)} \frac{k_{\text {in }}}{k_{\text {out }}}, B_{0}^{*}=\frac{2 \zeta_{0}}{\dot{Q}_{0}\left(i \zeta_{0}\right)} \frac{k_{\text {in }}}{k_{\text {out }}}
$$




$$
A_{2}^{*}=\frac{2 / 3}{P_{2}\left(i \zeta_{0}\right)-\frac{\dot{P}_{2}\left(i \zeta_{0}\right) Q_{2}\left(i \zeta_{0}\right)}{\dot{Q}_{2}\left(i \zeta_{0}\right)} \frac{k_{\text {in }}}{k_{\text {out }}}}, \quad B_{2}^{*}=B_{2}^{*} \frac{\dot{P}_{2}\left(i \zeta_{0}\right)}{\dot{Q}_{2}\left(i \zeta_{0}\right)} \frac{k_{\text {in }}}{k_{\text {out }}}
$$

The definitions of $\mathrm{P}_{\mathrm{n}}(\mathrm{i} \zeta)$ and $\mathrm{Q}_{\mathrm{n}}(\mathrm{i} \zeta)$ are also given in Lamb [19] and Chatjigeorhiou [20]. The dot over the $\mathrm{Q}_{2}, \mathrm{I}_{1}$ and $\mathrm{I}_{2}$ means differentiation with respect to $\zeta$.

\subsection{The induced velocity field}

The general solution for a triaxial ellipsoid was given by Miloh [15]. Here simplification for the lengthy expressions are sought for the prolate and oblate spheroids. The induced velocity can be written for the prolate spheroid as;

$$
\vec{u}=D_{T}\left[\sum_{k=1}^{3} G_{k} x_{k}^{2} \nabla I_{k}(\xi)-\nabla \Phi\right],
$$

where $\mathrm{D}_{\text {T }}$ represents the thermopheretic mobility and $\Phi$ is an harmonic function to be found along with the coefficients $G_{k}$. The subscript $k$ denotes the rectangular axis, where $k=1$ is the axis of symmetry. Because of axisymmetry $\mathrm{G}_{2}=\mathrm{G}_{3}$ and $\mathrm{I}_{2}=\mathrm{I}_{3}$. The functions $\mathrm{I}_{\mathrm{k}}$ are defined, following Miloh [15], as;

$$
I_{1}(\xi)=Q_{1}(\xi) / \xi, I_{2}(\xi)=Q_{1}^{1}(\xi) / P_{1}^{1}(\xi) \text {. }
$$

Eqs (17) and (18) can also hold for the oblate spheroid by simply replacing $\xi$ with ic. In order to show that Eq (17) indeed satisfies the continuity equation $(\nabla \cdot \vec{u}=0)$, all we need to show as already discussed in Miloh [15], is that $\nabla \cdot\left[x_{k}^{2} \nabla I_{k}(\xi)\right]=0 \quad$ (for any $\mathrm{k}=1,2,3$ ), since $\Phi$ in Eq (17) is harmonic. To prove this identity we first note that $x_{k}^{2} \partial I_{k} / \partial x_{i}=x_{k} \partial\left(x_{k} I_{k}\right) / \partial x_{i}-\delta_{u k} x_{k} I_{k}$ (no sum over $\mathrm{k}$ ), where $\delta_{u k}$ denotes the Kronecker symbol. Finally, the above divergence-free relation is easily verified by recalling that $x_{k} I_{k}(\xi)$ is also harmonic for $\mathrm{k}=1,2,3$ [15], leading to Eq (18).

The summation term on the right hand side of Eq (17) does not contribute to the slip velocity on the spheroid's surface. Hence $\vec{u}_{\text {slip }}=-D_{T} \nabla_{s} \Phi$, but the slip velocity is also linearly proportional to the surface temperature gradient by $\vec{u}_{\text {slip }}=-D_{T} \nabla_{s} T$. Therefore $\Phi$ is proportional to the temperature and can be shown to be;

$$
\Phi(\xi, \mu)=-\frac{q_{0} c^{2}}{6}\left[A_{2} P_{2}\left(\xi_{0}\right)-\frac{2}{3}\right] \frac{P_{2}(\mu) Q_{2}(\xi)}{Q_{2}\left(\xi_{0}\right)},
$$

for the prolate spheroid. Eq. (19) can still hold for the oblate spheroid by replacing $\xi$ with i $\zeta$ and $\mathrm{A}_{2}$ with $A_{2}^{*}$. The coefficients $G_{1}$ and $G_{2}$ can be found by imposing a zero normal velocity at the spheroid's surface. This procedure eventually leads to; 


$$
G_{1}=\frac{-q_{0}\left[A_{2} P_{2}\left(\xi_{0}\right)-\frac{2}{3}\right] \dot{Q}_{2}\left(\xi_{0}\right)}{6 \xi_{0}^{2} \dot{I}_{1}\left(\xi_{0}\right) Q_{2}\left(\xi_{0}\right)}, G_{2}=\frac{q_{0}\left[A_{2} P_{2}\left(\xi_{0}\right)-\frac{2}{3}\right] \dot{Q}_{2}\left(\xi_{0}\right)}{12 \xi_{0}^{2} \dot{I}_{2}\left(\xi_{0}\right) Q_{2}\left(\xi_{0}\right)},
$$

for the case of a prolate spheroid. Replacing $\xi$ with $i \xi$ and $A_{2}$ with $A^{*}{ }_{2}$ will yield the coefficients for the oblate spheroid.

\subsection{Numerical methodology}

The above analytical solutions were verified using numerical finite-difference schemes. The temperature field was directly calculated by solving Eqs (1) and (2) using a second order central differentiation scheme in both $(\xi, \mu)$ directions for the prolate spheroid and $(\zeta, \mu)$ directions for the oblate spheroid. Due to the symmetry conditions only a segment between the major and minor axes was solved as given in the contour plots of the next section, i.e. $0<\mu<1$. In order to avoid singularities at the major and minor axes as well as at the centre of the spheroid, mid-grid points were calculated where symmetry boundary conditions were used for the major and minor axes of the spheroid. First-order one-sided finite difference schemes were used to discretize the boundary condition matching the heat flux at the spheroid's surface. This approach prevents the generation of numerical wiggles in the solution due to the discontinuity in the temperature derivative at the spheroid's wall. Far from the spheroid the temperature was assumed to decay as $1 / \mathrm{r}$ where $\mathrm{r}$ is the radial distance from the spheroid's centre.

The discretization yielded a sparse matrix that was solved using the bi-stab and SOR algorithms [21]. A computational grid of $257^{2}$ points was found to be sufficient for the cases studied in Section 3 where the radial length of the computational domain is ten times of the equivalent radius $\mathrm{R}_{\text {equ}}$, i.e. the radius of a sphere with the same volume as of the spheroid. Excellent agreement was found between the analytical and numerical temperature solutions for all studied cases, verifying the analytical approach for the temperature field.

The velocity field can be numerically calculated using the stream-function vorticity formulation [22]. For the steady case, this involves simultaneously solving two elliptic equations for the stream function $\psi$ and the vorticity $\omega$. The two equations are coupled through the source of the streamfunction equation and the boundary condition on the spheroid's wall. The latter can be dealt through Toma's approach [22] but the coupling between the two equations will result in an iterative procedure that takes much longer than that for the temperature field. Since the numerical solution is needed only in order to verify the analytical solution, a somewhat simpler approach was used for this study. The induced velocity field was calculated according to Eq (17) and then was verified to 
fulfil the continuity and momentum equations. This means $\nabla \cdot \vec{u}=0, \nabla \times \nabla \times \vec{\omega}=0$, where the equation for the vorticity accounts for the fact that the spheroidal coordinates system is not rectangular but is still orthonormal. Both equations were found to be fulfilled down to the truncation errors of the operators that were calculated using second-order finite-difference central schemes.

\section{Results and discussion}

Prolate and oblate spheroids with an equivalent radius $R_{\text {equ }}=1 \mu \mathrm{m}$ were investigated for aspect ratios of $\mathrm{D} / \mathrm{d}=2$ to 4 for the prolates and the inverse aspect ratios for the oblates. The surrounding medium's thermal conductivity was set to $\mathrm{k}_{\mathrm{out}}=0.6 \mathrm{~W} /(\mathrm{m} \mathrm{K})$ as of fresh water. The spheroid's thermal conductivity was taken as $k_{\text {in }}=0.04 \mathrm{~W} /(\mathrm{m} \mathrm{K})$ or $8 \mathrm{~W} /(\mathrm{m} \mathrm{K})$. The lower value of $\mathrm{k}_{\text {in }}$ corresponds to that of polystyrene and the higher value corresponds to that of Beryllium copper. This means that for the polystyrene spheroid, the surrounding water acts as a heat sink while for the Beryllium copper spheroid the surrounding water acts as an insulator. The uniform heat density $\mathrm{q}_{0}$ inside the spheroid was taken as $10^{12} \mathrm{~W} / \mathrm{m}^{3}$ for all cases.

\subsection{The temperature and heat flux fields}

Table 1 lists the temperature at the centre of the spheroid, which is also the maximum temperature and the averaged surface temperature. It is seen that increasing $k_{\text {in }}$ from $0.04 \mathrm{~W} /(\mathrm{m} \mathrm{K})$ to $8 \mathrm{~W} /(\mathrm{m} \mathrm{K})$ caused the maximum temperature to considerably decrease. This behaviour can also be seen from the spherical analytical solution of Eq (5). Increasing $\mathrm{k}_{\mathrm{in}}$ means the particle becomes a better conductor and thus more heat is lost to the surrounding medium.

On the other hand, the averaged surface temperature is much less affected by the value of $\mathrm{k}_{\text {in }}$. Increasing the spheroid aspect-ratio $\mathrm{D} / \mathrm{d}$ for the prolate or decreasing it for the oblate, causes the averaged surface temperature to decrease. This is because as the ratio $\mathrm{D} / \mathrm{d}$ is shifted away from one making the spheroid's less spherical, the surface area of the spheroid increases, while the volume of the spheroid is kept the same. Hence, more heat can be lost through the surface for the same overall heat source of $\widetilde{Q}_{0}=4 q_{0} \pi R^{3} / 3$. Despite the small variation of the averaged surface temperature with the aspect ratio D/d, excellent agreement was found with Baffou's 'fitting' thermal expression given in Miloh [15].

A graphical presentation of the temperature variations along the spheroid's major and minor axes is given for all studied cases in Fig 2. The spherical solution of Eqs (6) and (7) is also shown for the equivalent sphere. All spheroids show maximum temperatures that are lower than that of the sphere. Again, this is because the sphere has the smallest ratio of surface area to volume. The spheroids 
corresponding to $\mathrm{k}_{\text {in }}=0.04 \mathrm{~W} /(\mathrm{m} \mathrm{K})$ show much steeper variation in the temperature inside the spheroid than that of $\mathrm{k}_{\mathrm{in}}=8 \mathrm{~W} /(\mathrm{m} \mathrm{K})$. It is a result of the better insulation caused by the lower value of $\mathrm{k}_{\mathrm{in}}$, managing to keep a higher temperature around the centre of the spheroid.

All spheroids also show that the farthest tip has a temperature lower than of the nearest tip. This means that for the prolate spheroid the temperature at the major axis tip is lower than that at the minor axis tip and vice versa for the oblate spheroid. The tips can be recognized in Fig 2 as the points of discontinuity in the first derivative of the temperature distribution. The effect of $k_{\text {in }}$ diminishes as one gets away from the spheroid, where the temperature decay converges to the spherical solution of Eq (6). However, unlike the spherical case where $k_{\text {in }}$ has no role at all in the temperature field $\mathrm{T}_{\text {out }}$ outside the particle, $\mathrm{k}_{\text {in }}$ affects $\mathrm{T}_{\text {out }}$ near the particle and its decay is not as of spherical. This zone just outside the spheroid will be called the near field of $\mathrm{T}_{\text {out }}$ while the farther zone where $\mathrm{T}_{\text {out }}$ converges to the spherical solution will be called the far field.

Illustrations of the contours of the temperature field are given in Fig 3 for the prolate spheroid of $\mathrm{D} / \mathrm{d}=4$ and oblate spheroid of $\mathrm{D} / \mathrm{d}=1 / 4$. The contour lines follow loosely lines parallel to the spheroid's geometry, where the difference between the far field $\mathrm{T}_{\text {out }}$ and the spheroid's temperature is much smaller for $k_{\text {in }}=8 \mathrm{~W} /(\mathrm{m} \mathrm{K})$ than for $\mathrm{k}_{\mathrm{in}}=0.04 \mathrm{~W} /(\mathrm{m} \mathrm{K})$ as already seen in Fig 2. The temperature $\mathrm{T}_{\text {out }}$ along the major axis of the prolate spheroid shows a weaker decay than that along the minor axis, pointing again to the effect of the near field of $\mathrm{T}_{\text {out }}$ that does not exist in the spherical case. A similar behaviour is seen for the oblate spheroid where the more moderate temperature decay is at the minor axis instead of the major axis.

The variations of the heat flux along the major and minor axes of the prolate and oblate spheroids are shown in Fig 4. As expected, the peak of the heat flux's distribution along the axis is always at the spheroid's tip, where there is a discontinuity in the first derivative of the heat flux due to the difference between $k_{\text {in }}$ and $k_{\text {out }}$. The ratio of $k_{\text {in }} / k_{\text {out }}$ affects whether the maximum of the heat flux is at the spheroid's tip that is nearest to the centre or at the tip that is farthest. For $k_{\text {in }} / k_{\text {out }}=0.0667$, i.e. the surrounding acts as a heat sink, the maximum value of the heat flux is at the tip nearest to the centre, i.e. at the minor axis for the prolate spheroid and at the major axis for the oblate spheroid. For $\mathrm{k}_{\mathrm{in}} / \mathrm{k}_{\text {out }}=13.333$ the situation is opposite and the maximum value for the heat flux is at the farthest tip of the spheroid, whether it is a prolate or an oblate.

The effect of the ratio $\mathrm{k}_{\text {in }} / \mathrm{k}_{\text {out }}$ is further illustrated in the contour plots of Fig 5 for the heat flux around the prolate spheroid with an aspect ratio of $\mathrm{D} / \mathrm{d}=4$. The minor axis tip area clearly shows the 
maximum of the heat flux for the case of $\mathrm{k}_{\mathrm{in}}=0.04 \mathrm{~W} /(\mathrm{m} \mathrm{K})$, while the major axis tip area shows the maximum of the heat flux for the case of $\mathrm{k}_{\text {in }}=8 \mathrm{~W} /(\mathrm{m} \mathrm{K})$. The shift in the location of the maximum heat flux from the nearest spheroid's tip to the farthest when $k_{\text {in }}$ was increased from 0.04 $\mathrm{W} /(\mathrm{m} \mathrm{K})$ to $8 \mathrm{~W} /(\mathrm{m} \mathrm{K})$ is related to the behaviour of the near field of $\mathrm{T}_{\text {out }}$ This is because the maximum temperature on the spheroid's surface stays at the nearest tip regardless of the change in the value of $k_{\text {in }}$ and hence if the far field with its $1 / \mathrm{r}$ decay in $T_{\text {out }}$ had started straight from the spheroid's surface, a simple arithmetic operation shows that the maximum heat flux would have stayed at the location where the maximum surface temperature is located, i.e. at the tip nearest to the spheroid's centre. Examining again the temperature variations along the major and minor axes in Fig 2, one can see that for example for the prolate spheroid in Figs $2 \mathrm{a}$ and $2 \mathrm{~b}$, increasing $\mathrm{k}_{\text {in }}$ from $0.04 \mathrm{~W} /(\mathrm{m} \mathrm{K})$ to $8 \mathrm{~W} /(\mathrm{m} \mathrm{K})$ causes the temperature at the farthest tip to increase and to yield a steeper decline of $\mathrm{T}_{\text {out }}$ in the near field. This results in a sharp increase in the heat flux at that tip as seen in the corresponding Figs 4a and 4b. Similar behaviour occurs in the oblate spheroid case.

\subsection{The induced velocity field in the surrounding medium}

The induced velocity vectors are shown in Figs 6 and 7 for the prolate and oblate spheroids respectively. For simplicity $\mathrm{D}_{\text {T }}$ was set as $1 \mathrm{~m}^{2} /(\mathrm{s} \mathrm{K})$. Any other value will just adjust the magnitude of the velocity vector due to the linearity of the problem. In all cases the velocity pattern resembles that of stagnation flow around a corner except that the maximum velocity magnitude is at the spheroid's surface and not far from its farthest tip. Because the temperature at the spheroid's nearest tip is always higher than that at the farthest tip, the slip flow is always from the nearest tip to the farthest tip.

Increasing $\mathrm{k}_{\text {in }}$ from $0.04 \mathrm{~W} /(\mathrm{m} \mathrm{K})$ to $8 \mathrm{~W} /(\mathrm{m} \mathrm{K})$ significantly reduced the velocity magnitude as clearly seen in Figs 6 and 7. This is because of the more uniform temperature over the spheroid's surface as can be seen in Figs 2 and 3. It results in a lower surface temperature gradient $\nabla_{s} T$ and thus a lower magnitude for the induced slip velocity. On the other hand, it was found that increasing the aspect ratio of $\mathrm{D} / \mathrm{d}$ for the prolate spheroid or decreasing it for the oblate spheroid makes the spheroid less spherical and thus increases the temperature variation along the surface. This yields a higher surface temperature gradient and hence a higher induced velocity.

The vorticity contours are shown in Fig 8 for the prolate spheroid of $D / d=4$. The vorticity was numerically calculated from the velocity field using a second order central finite-difference scheme and the appropriate scale factors h's for the spheroidal coordinates. In both cases the vorticity peaks at the spheroid's wall near the farthest tip, but not exactly at the farthest tip due to the symmetry 
condition at that axis. Similar behaviour was found for all other prolate and oblate spheroids. This peak of the vorticity affects the distribution of the stream function as shown in Fig 9 for the cases corresponding to Fig 8. The stream function $\psi$ was calculated according to the following equation for the prolate system where the appropriate h's were used [7];

$$
-\omega=\frac{1}{h_{1} h_{2}}\left[\frac{\partial}{\partial \xi}\left(\frac{h_{2}}{h_{1} h_{3}} \frac{\partial \psi}{\partial \xi}\right)+\left(\frac{h_{1}}{h_{2} h_{3}} \frac{\partial \psi}{\partial \mu}\right)\right] .
$$

The same equation can be used for the oblate case by replacing $\xi$ with $\zeta$ and using the appropriate h’s for the oblate spheroidal coordinates system [7].

The stream-function pattern in Fig 9 is similar to that of flow around a corner. The highest density of the streamlines is at the area of the vorticity's peak near the spheroid's farthest tip, showing it to be corresponding to the maximum velocity magnitude. Similar behaviour was found for all other studied spheroidal configurations. Fig 9 shows the stream-function field up to $10 \mathrm{R}_{\text {equ }}$ away from the spheroid's centre. Further away into the far field the streamlines converge into close patterns although the velocity magnitude is very small. Hence, for the prolate spheroid the flow goes down along the minor axis towards the spheroid until hitting its nearest tip. The flow slips over the spheroid's surface to its farthest tip and then flows at the positive direction of the major axis away from the spheroid. The flow turns up in the far field while losing magnitude until it flows back towards the spheroid in the negative direction of the minor axis. Similar pattern occurs for the oblate spheroid, but just the roles of the minor and major axes are switched.

\section{Conclusion}

A micro/nano conducting orthotropic (spheroidal) particle uniformly heated by light (laser) and embedded in a uniform low-conducting (liquid) medium, was studied for its temperature variations and induced velocity field driven by the Soret surface slip-velocity. The temperature fields in both phases are governed by the Poisson and Laplace linearized heat equations and the velocity field is assumed to be of Stokes flow.

Analytical solutions were derived under Rayleigh's assumption of even heating for the temperature and induced velocity fields using Legendre series. The temperature solution depended only on two modes of the series. These analytical solutions were verified using numerical finite-difference schemes.

As expected, the spheroid's maximum temperature lies always at its centre. The maximum of the spheroid's surface temperature was at the tip closest to the centre for all studied cases. The 
maximum magnitude of the heat flux was at the spheroid's surface. The peak of the heat flux was at the spheroid's tip nearest to the centre for $k_{\text {in }} / k_{\text {out }}<1$ and at the farthest tip for $k_{\text {in }} / k_{\text {out }}>1$. The change in the behaviour of the heat flux was related to the creation of a near field of the surrounding temperature $\mathrm{T}_{\text {out }}$ that showed a temperature decay different than in the far field, which converged to the spherical solution decay of $1 / r$.

The induced flow field was shown to flow from spheroid's nearest tip to the farthest tip, where increasing the spheroid aspect ratio for the prolate configuration or reducing the aspect ratio for the oblate configuration, increased the magnitude of the induced velocity field. The amplitude of the vorticity peaked (maximum mixing) on the spheroid's surface and near the farthest tip. Further away from the spheroid, the flow direction (quadrupole nature) was towards the spheroid's nearest tip and away from the spheroid's farthest tip where the flow re-circled in the far field from the farthest tip's axis to the nearest tip's axis.

The model problem considered here is indeed ideal in terms of assuming uniform (constant) thermal conductivities and heat density, neglecting the effects of surface resistivity, nearby substrates and particles but nevertheless can be used to get good estimates of the relevant thermal and hydro-dynamical parameters. For more complex geometries a full numerical approach is probably needed and further experimental studies in this growing field of research interest are certainly welcome.

Acknowledgment: This work was partially supported by ISF Grant 1945/14.

\section{References:}

[1] MA Rahman and MZ Sagir, Thermodiffusion or Soret effect: Historical review, Int J Heat Mass Trans 73 (2014) 693-705

[2] F Zheng, Thermophoresis of spherical and non-spherical particles: a review of theories and experiments, Adv Colloid Interfac Sci 97 (2002) 255-278

[3] HJ Keh and YC Chang, Thermophoresis of an aerosol spheroid along its axis of revolution, Phys Fluids 21, (2009) 062001

[4] T Miloh and J Nagier, Light-induced self-thermophoresis of Janus spheroidal nanoparticles, Electrophoresis 39 (2018) 2417-2424

[5] HR Jiang, N Yoshinaga and M Sano, Active motion of a Janus particle by self-thermophoresis in a defocused laser beam, Phys Rev Lett 105 (2010) 268302 
[6] H Moyses, J Palacci, S Sacanna and DG Grier, Trochoidal trajectories of self-propelled Janus particles in a diverging laser beam, Soft Matter 12 (2016) 6357-6364

[7] HH Wei and JS Jan, Self-propulsion and dispersion of reactive colloids due to entropic anisotropy, J Fluid Mech 657 (2010) 64-88

[8] G Baffou, Thermoplasmonics, Heating metal nanoparticles using Light, Cambridge Univ Press, Cambridge, 2018

[9] AO Govorov and HH Richardson, Generating heat with metal nanoparticles, nanotoday 2 (2007) 30-38

[10] HH Richardson, MT Carlson, PJ Tandler P Hernandez and AO Govorov, Experimental and theoretical studies of light-to-heat conversion and collective heating effects in metal nanoparticle solutions, Nano Lett 9 (2009) 1139-1146

[11] VK Pustovalov, Light-to heat conversion and heating of single nanoparticles, their assemblies, and the surrounding medium under laser pulses, RSC Adv 6 (2016) 81266-81289

[12] D Jaque, LM Masetro, D del Rosal, P Haro-Gonzalez, A Benayas, JL Plaza EM Rodriguez and JG Sole, Nanoparticles for photothermal therapies, Nanoscale 6 (2014) 9494-9530

[13] Z Wang and P. Cheng. Enhancement of absorption and photothermal conversion of solar energy enable by surface plasmon resonances in nanoparticles and metamaterials. Int. J. Heat \& Mass Transfer 140 (2019), 453-482.

[14] D. Wang, P. Cheng and X. Quan. Photothermal nanobubble nucleation on a plasmonic nanoparticle: A 3D lattice Boltzmann simulations. Int. J. Heat \& Mass Transfer 140 (2019), 786797.

[15] T Miloh, Light-induced thermoosmosis about conducting ellipsoidal nano particles, Proc Royal Soc A, 475 (2019), 20180040.

[16] H Brenner, Beyond the no-slip boundary condition, Phys Rev E 84 (2011) 046309

[17] A Wurger. Thermal non-equilibrium transport in colloids. Reports on Progress in Physics. 73 (2010), 126601.

[18] R Ganti, Y Liu and D Frenkel, Molecular simulation of thermo-osmotic slip, Phys Rev Lett 119 (2017) 038002

[19] H Lamb, Hydrodynamics, Dover Pub, New York, 1945

[20] IK Chatjigeorhiou, Analytical methods in marine hydrodynamics, Cambridge Univ Press, Cambridge, 2018

[21] KM Singh, EJ Avital, JJR Williams, C Ji, X Bai, A Munjiza, On parallel pre-conditioners for pressure Poisson equation in LES of complex geometry flows, Int J Num Meth Fluids 83(5) (2017), 446-464 
[22] EJ Avital, ND Sandham, KH Luo and RE Musafir, Calculation of basic sound radiation of axisymmetric jets by direct numerical simulations, AIAA J., 37(2), (1999), 161-168 


\begin{tabular}{|c|c|c|c|}
\hline Spheroid geometric ratio & $\mathrm{k}_{\mathrm{in}}[\mathrm{W} /(\mathrm{m} \mathrm{K})]$ & $\mathrm{T}_{\max }[\mathrm{K}]$ & $\mathrm{T}_{\text {av }}$ on spheroid surface $[\mathrm{K}]$ \\
\hline 1 (sphere) & 0.04 & 4.722 & 0.5550 \\
\hline 2 (prolate) & 0.04 & 4.0748 & 0.5406 \\
\hline 4 (prolate) & 0.04 & 2.9614 & 0.4902 \\
\hline $1 / 2$ (oblate) & 0.04 & 3.9150 & 0.5425 \\
\hline $1 / 4$ (oblate) & 0.04 & 2.4031 & 0.5148 \\
\hline 1 (sphere) & 8 & 0.5764 & 0.5550 \\
\hline 2 (prolate) & 8 & 0.5570 & 0.5338 \\
\hline 4 (prolate) & 8 & 0.5082 & 0.4761 \\
\hline $1 / 2$ (oblate) & 8 & 0.5572 & 0.5347 \\
\hline $1 / 4$ (oblate) & 8 & 0.5090 & 0.4817 \\
\hline
\end{tabular}

Table 1: The computed values of the maximum temperature inside the spheroid (i.e. at the centre) and the average temperature on the spheroid's surface, where $\mathrm{k}_{\text {out }}$ is $0.6 \mathrm{~W} /(\mathrm{m} \mathrm{K})$. 


\section{List of Figures:}

Figure 1: Schematic description of the problem for a prolate spheroidal particle.

Figure 2: The temperature variations along the major and minor axes that are plotted for the prolate spheroids of (a) $D / d=2$ and (b) $D / d=4$, and the oblate spheroids of (c) $D / d=1 / 2$ and (d) $D / d=1 / 4$. The external thermal conductivity is $\mathrm{k}_{\mathrm{e}}=0.6 \mathrm{~W} /(\mathrm{m} \mathrm{K})$. The distance from the spheroid's centre noted as the horizontal axis is normalized by the radius $R_{\text {equ }}$ of the equivalent sphere, whose solution was also added.

Figure 3: The temperature contours in $\mathrm{K}$ for the prolate spheroid of $\mathrm{D} / \mathrm{d}=4$ and where the spheroid's thermal conductivity is (a) $k_{i}=0.04 \mathrm{~W} /(\mathrm{m} \mathrm{K})$ and (b) $k_{i}=8 \mathrm{~W} /(\mathrm{m} \mathrm{K})$. The rest of the conditions are as in Fig. 2.

Figure 4: The heat flux magnitude variations along the major and minor axes of the prolate spheroids (a) $(D / d)=2$ and (b) $(D / d)=4$, and the oblate spheroids (c) $(D / d)=1 / 2$ and $(d)(D / d)=1 / 4$, and where the rest of the conditions are as in Fig. 2.

Figure 5: The heat flux contours in in $\mathrm{kW} / \mathrm{m}^{2}$ for the prolate spheroid of $\mathrm{D} / \mathrm{d}=4$ and where the spheroid's thermal conductivity is (a) $k_{i}=0.04 \mathrm{~W} /\left(\mathrm{m} \mathrm{K}\right.$ ) and (b) $k_{i}=8 \mathrm{~W} /(\mathrm{m} \mathrm{K})$. The rest of the conditions are as in Fig. 2.

Figure 6: Induced velocity vectors that are plotted for the prolate spheroid of $D / d=4$ and where the spheroid's thermal conductivity is (a) $\mathrm{k}_{\mathrm{i}}=0.04 \mathrm{~W} /(\mathrm{m} \mathrm{K})$ and (b) $\mathrm{k}_{\mathrm{i}}=8 \mathrm{~W} /(\mathrm{m} \mathrm{K})$. The vector magnitude has been increased by $10 / 3$ for $\mathrm{k}_{\mathrm{i}}=8 \mathrm{~W} /(\mathrm{m} \mathrm{K})$ as compared to $\mathrm{k}_{\mathrm{i}}=0.04 \mathrm{~W} /(\mathrm{m} \mathrm{K})$. The rest of the conditions are as in Fig 2.

Figure 7: Induced velocity vectors that are plotted for the oblate spheroid of $D / d=1 / 4$ and where the spheroid's thermal conductivity is (a) $\mathrm{k}_{\mathrm{i}}=0.04 \mathrm{~W} /(\mathrm{m} \mathrm{K})$ and (b) $\mathrm{k}_{\mathrm{i}}=8 \mathrm{~W} /(\mathrm{m} \mathrm{K})$. The vector magnitude has been increased by $10 / 3$ for $\mathrm{k}_{\mathrm{i}}=8 \mathrm{~W} /(\mathrm{m} \mathrm{K})$ as compared to $\mathrm{k}_{\mathrm{i}}=0.04 \mathrm{~W} /(\mathrm{m} \mathrm{K})$. The rest of the conditions are as in Fig 2.

Figure 8: Contours of the vorticity in $1 / \mathrm{s}$ that are plotted for the prolate spheroids (a) $D / d=4$ and $\mathrm{k}_{\mathrm{i}}=0.04 \mathrm{~W} /(\mathrm{m} \mathrm{K})$, (b) $\mathrm{D} / \mathrm{d}=4$ and $\mathrm{k}_{\mathrm{i}}=8 \mathrm{~W} /(\mathrm{m} \mathrm{K})$. The rest of the conditions are as in Fig 2.

Figure 9: Contours of the stream-function in $\mathrm{m}^{2} / \mathrm{s}$ that are plotted for the prolate spheroids (a) $\mathrm{D} / \mathrm{d}=4$ and $\mathrm{k}_{\mathrm{i}}=0.04 \mathrm{~W} /(\mathrm{m} \mathrm{K})$, (b) $\mathrm{D} / \mathrm{d}=4$ and $\mathrm{k}_{\mathrm{i}}=8 \mathrm{~W} /(\mathrm{m} \mathrm{K})$. The rest of the conditions are as in Fig 2. 


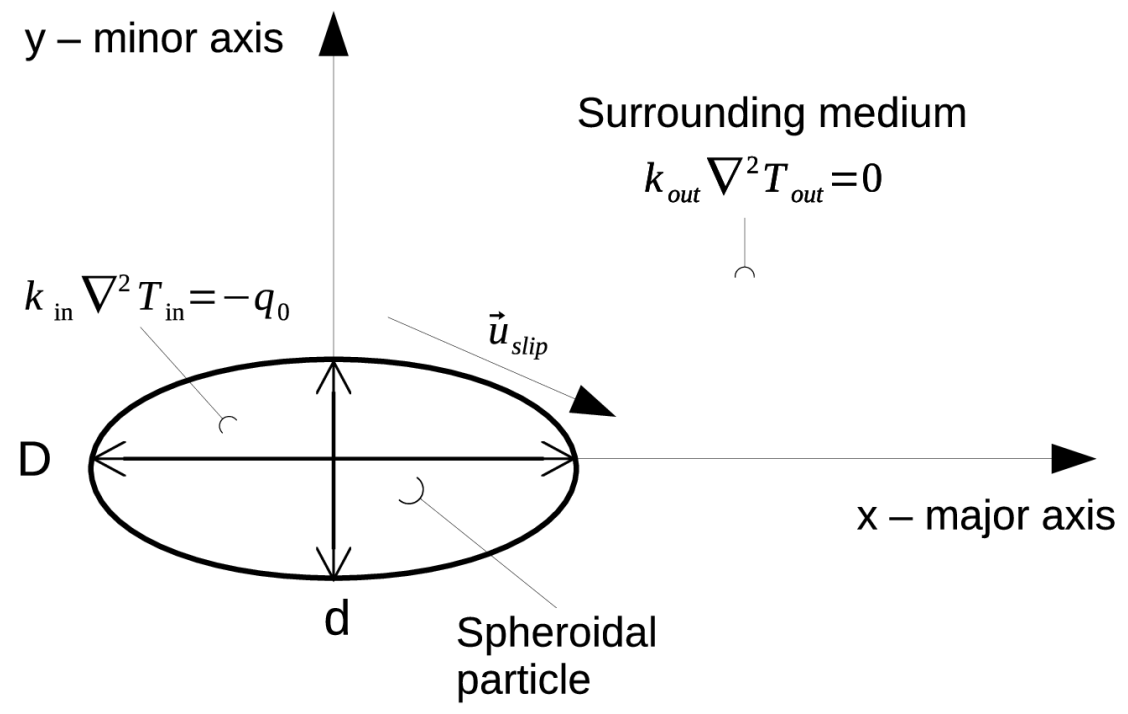

Figure 1: Schematic description of the problem for a prolate spheroidal particle. 
(a)

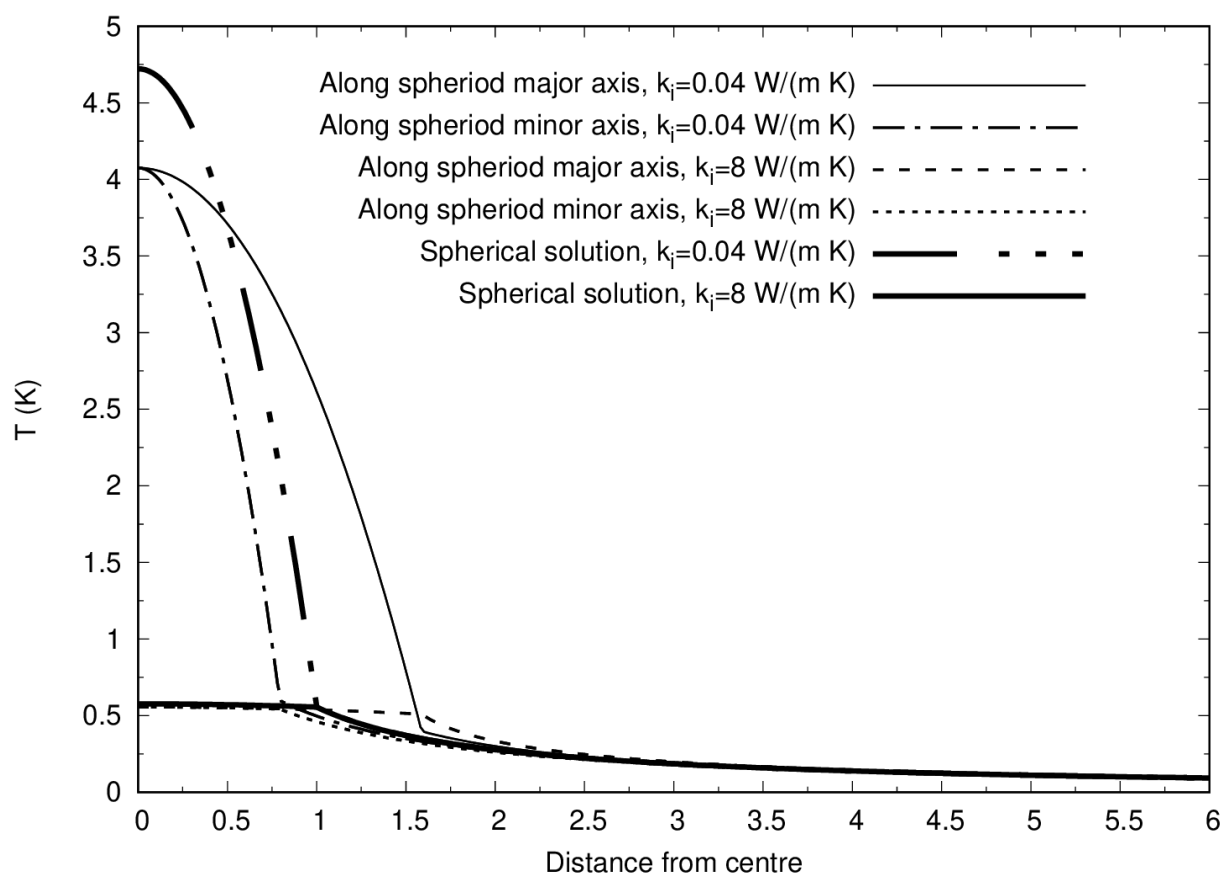

(b)

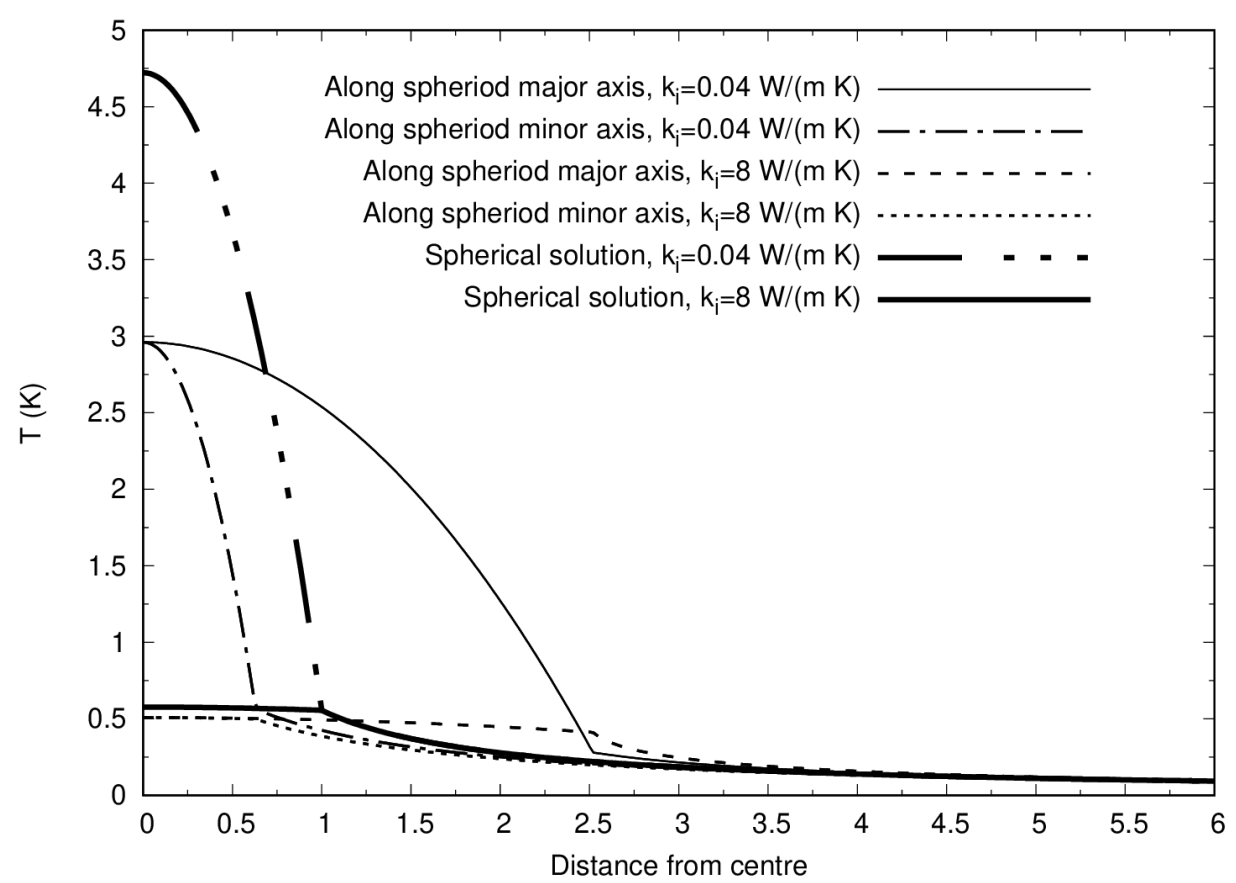


(c)

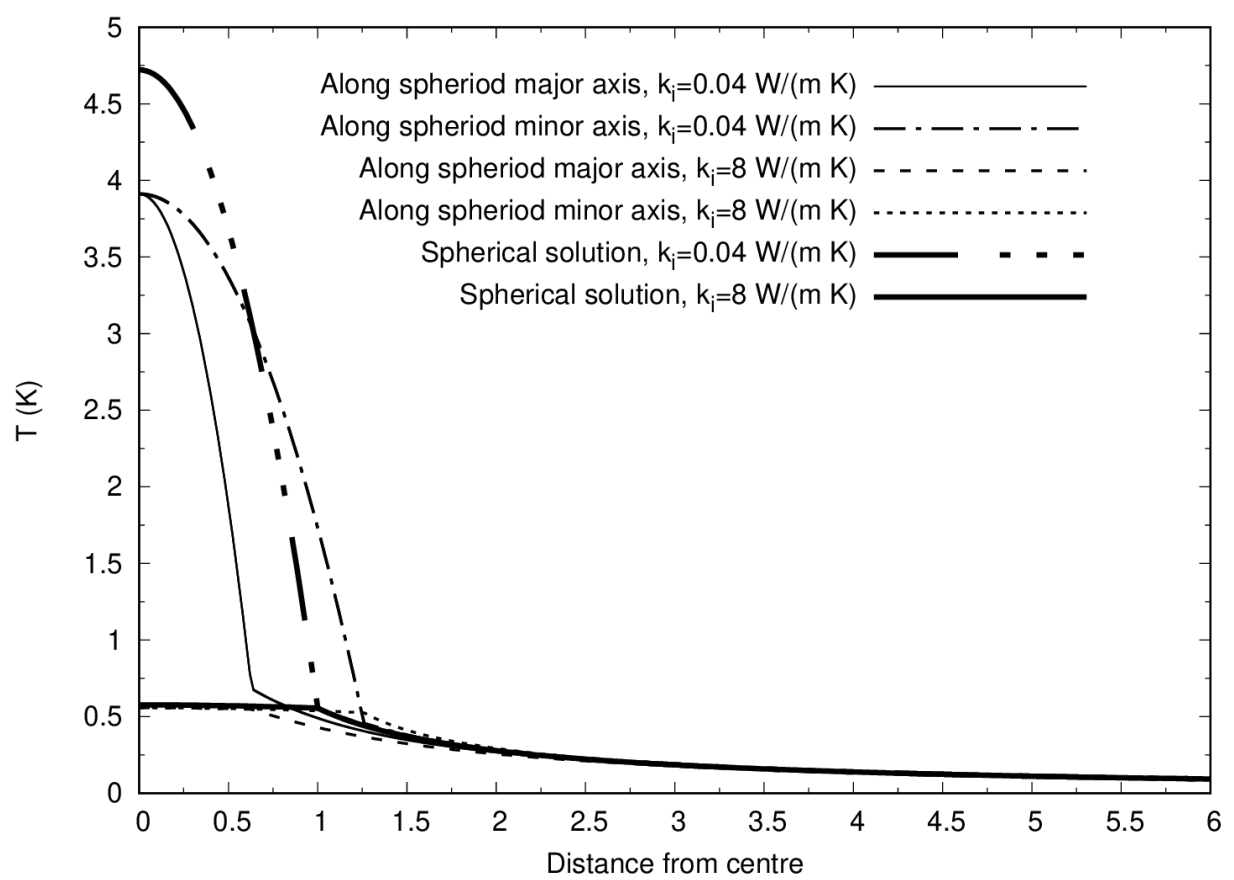

(d)

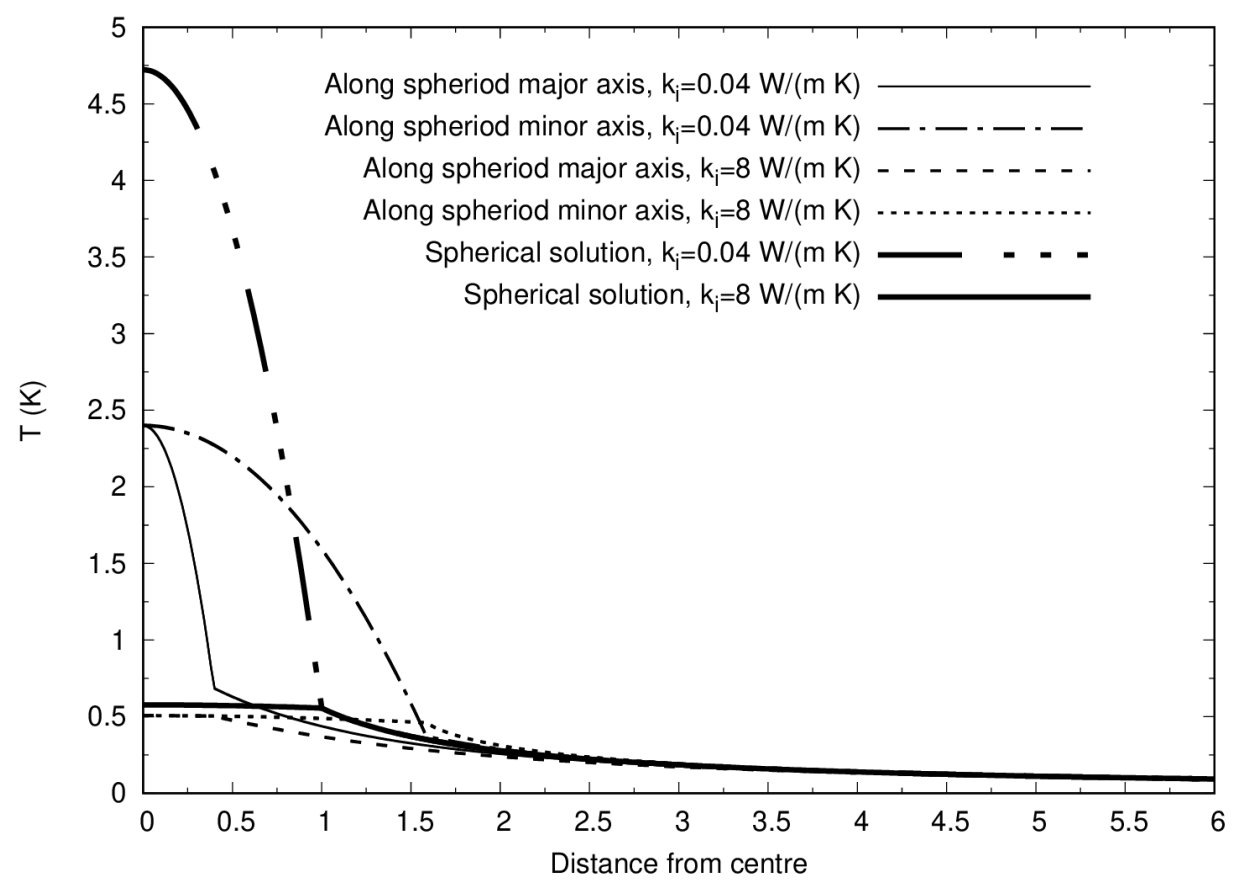

Figure 2: The temperature variations along the major and minor axes that are plotted for the prolate spheroids of (a) $D / d=2$ and (b) $D / d=4$, and the oblate spheroids of (c) $D / d=1 / 2$ and (d) $D / d=1 / 4$. The external thermal conductivity is $\mathrm{k}_{\mathrm{e}}=0.6 \mathrm{~W} /(\mathrm{m} \mathrm{K})$. The distance from the spheroid's centre noted as the horizontal axis is normalized by the radius $R_{\text {equ }}$ of the equivalent sphere, whose solution was also added. 
(a)

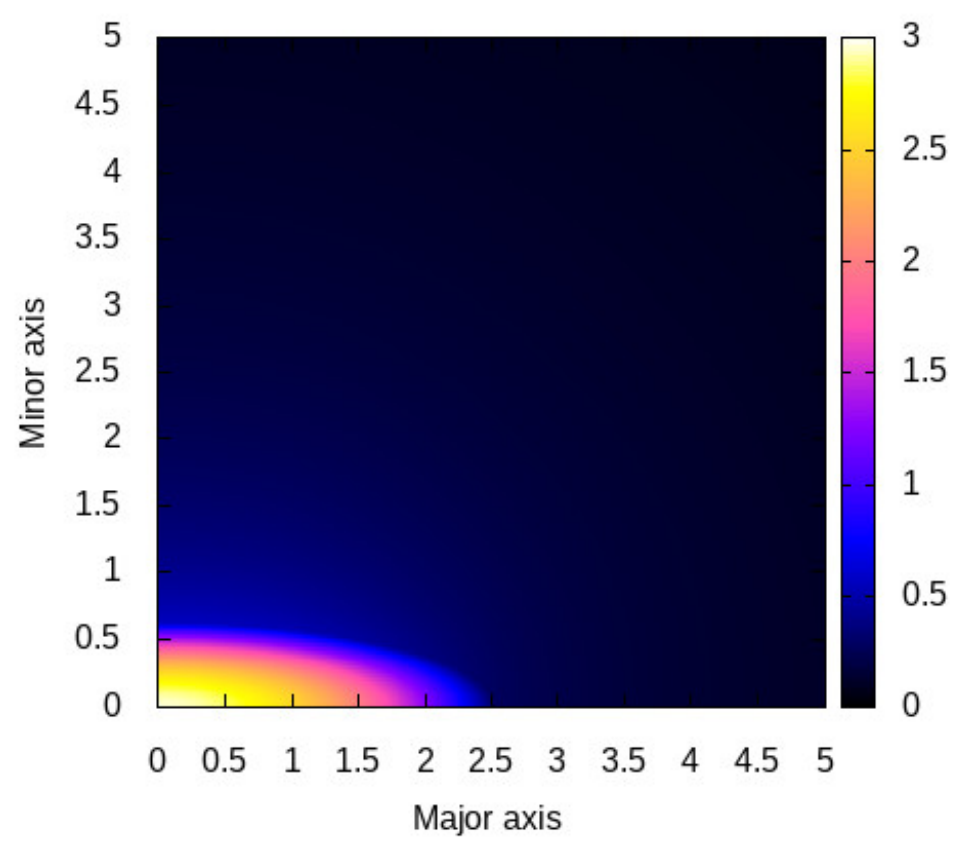

(b)

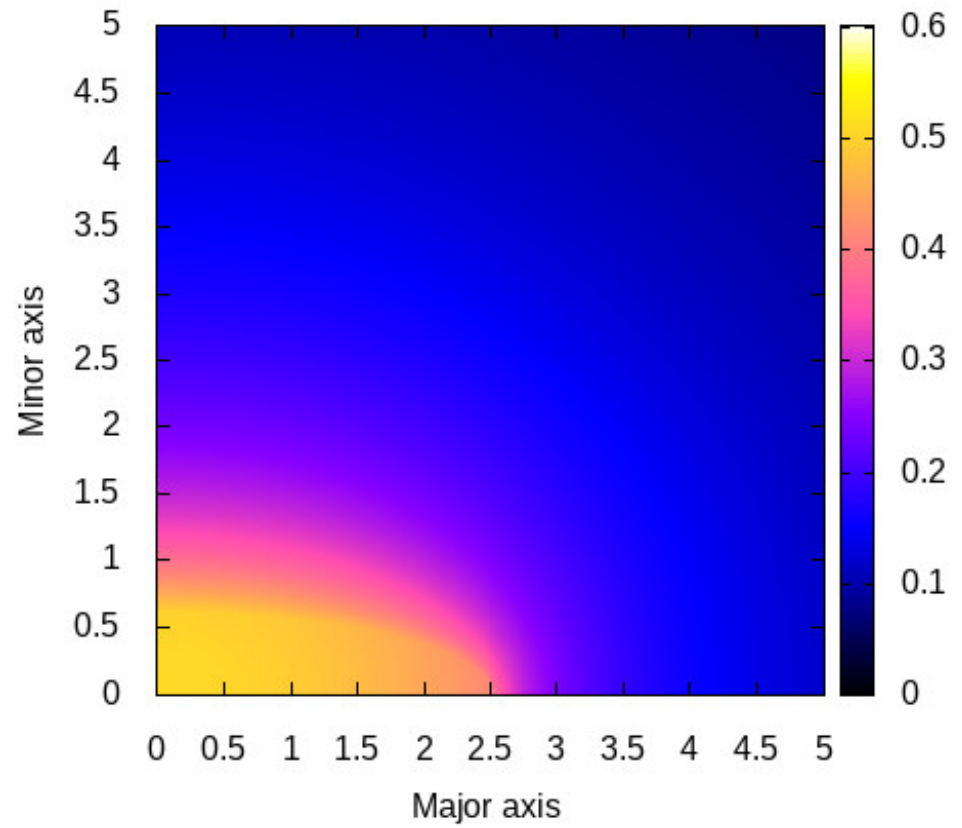

Figure 3: The temperature contours in $\mathrm{K}$ for the prolate spheroid of $\mathrm{D} / \mathrm{d}=4$ and where the spheroid's thermal conductivity is (a) $\mathrm{k}_{\mathrm{i}}=0.04 \mathrm{~W} /(\mathrm{m} \mathrm{K})$ and (b) $\mathrm{k}_{\mathrm{i}}=8 \mathrm{~W} /(\mathrm{m} \mathrm{K})$. The rest of the conditions are as in Fig. 2. 
(a)

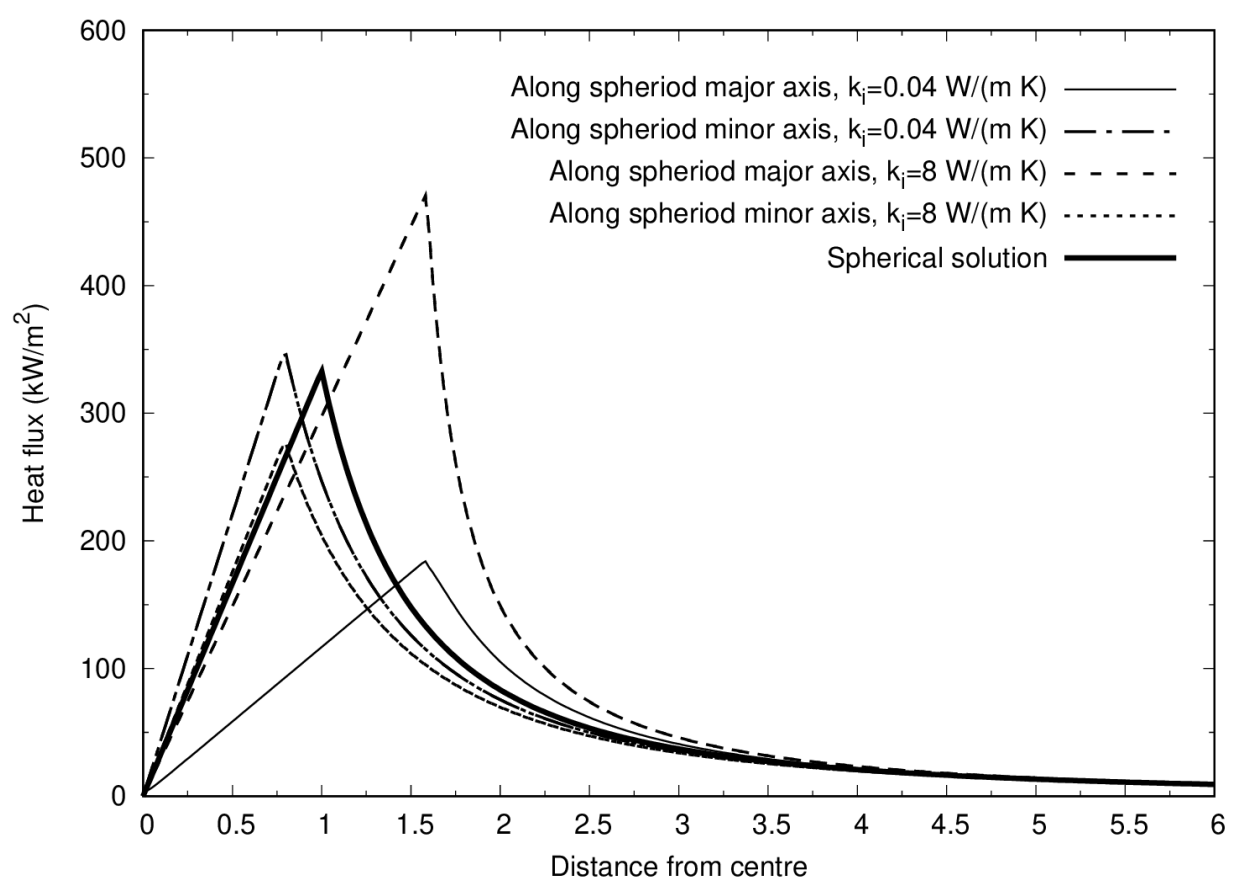

(b)

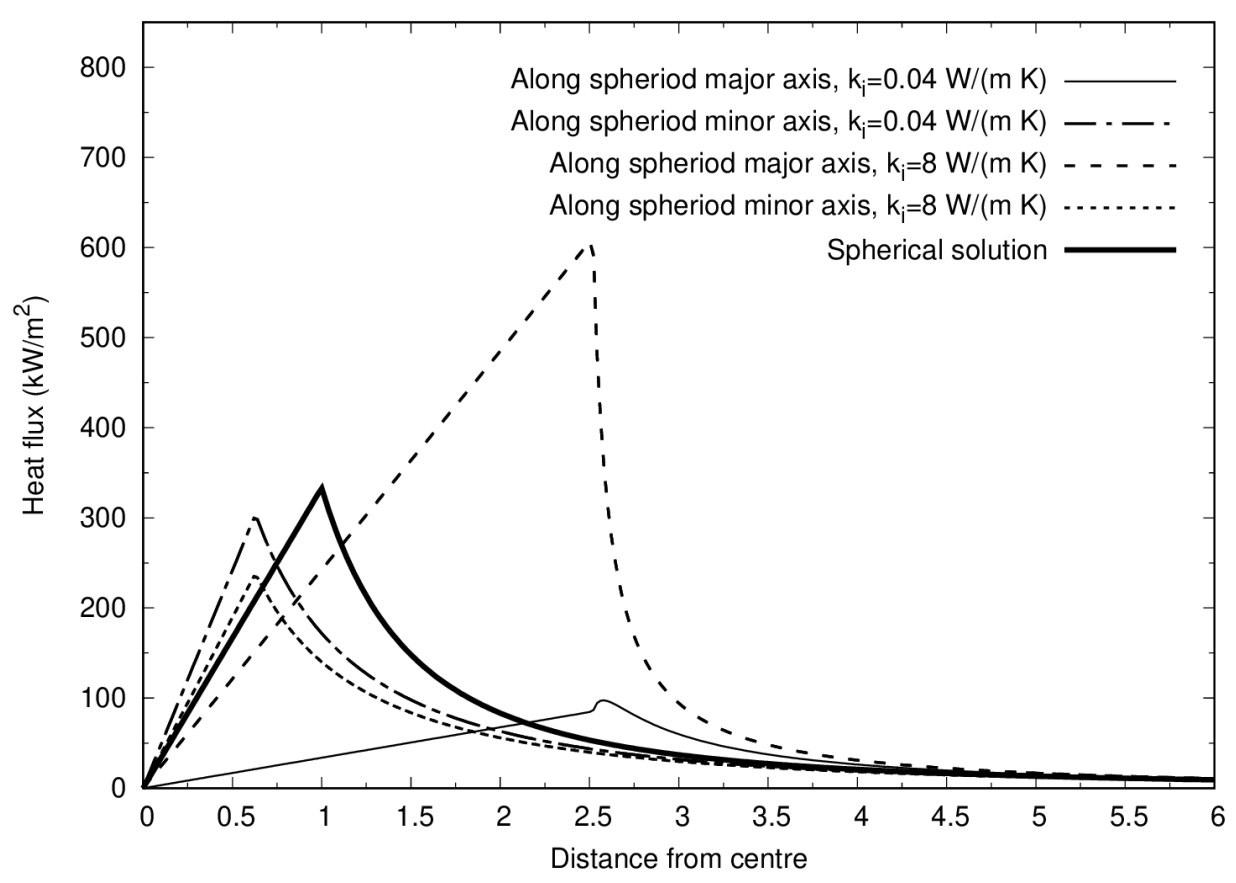


(c)

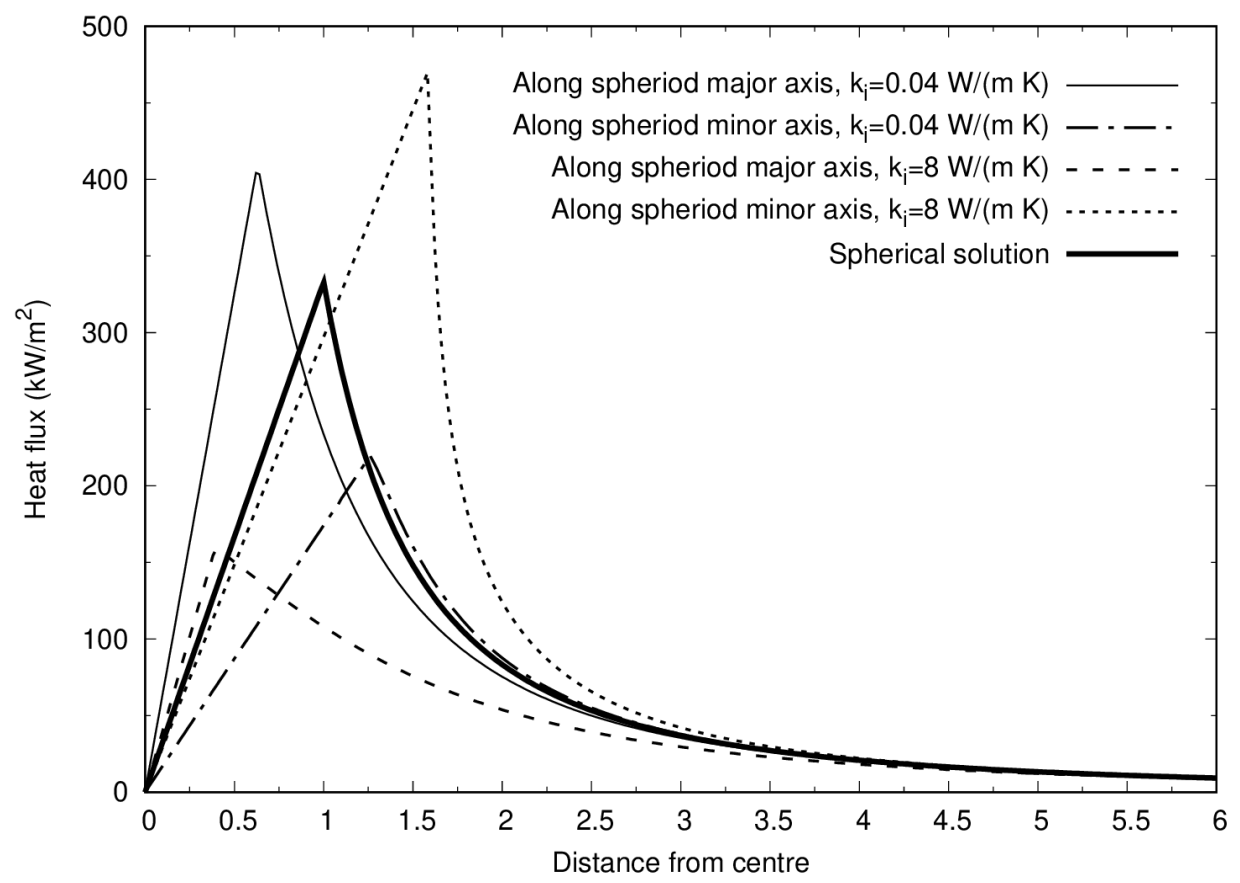

(d)

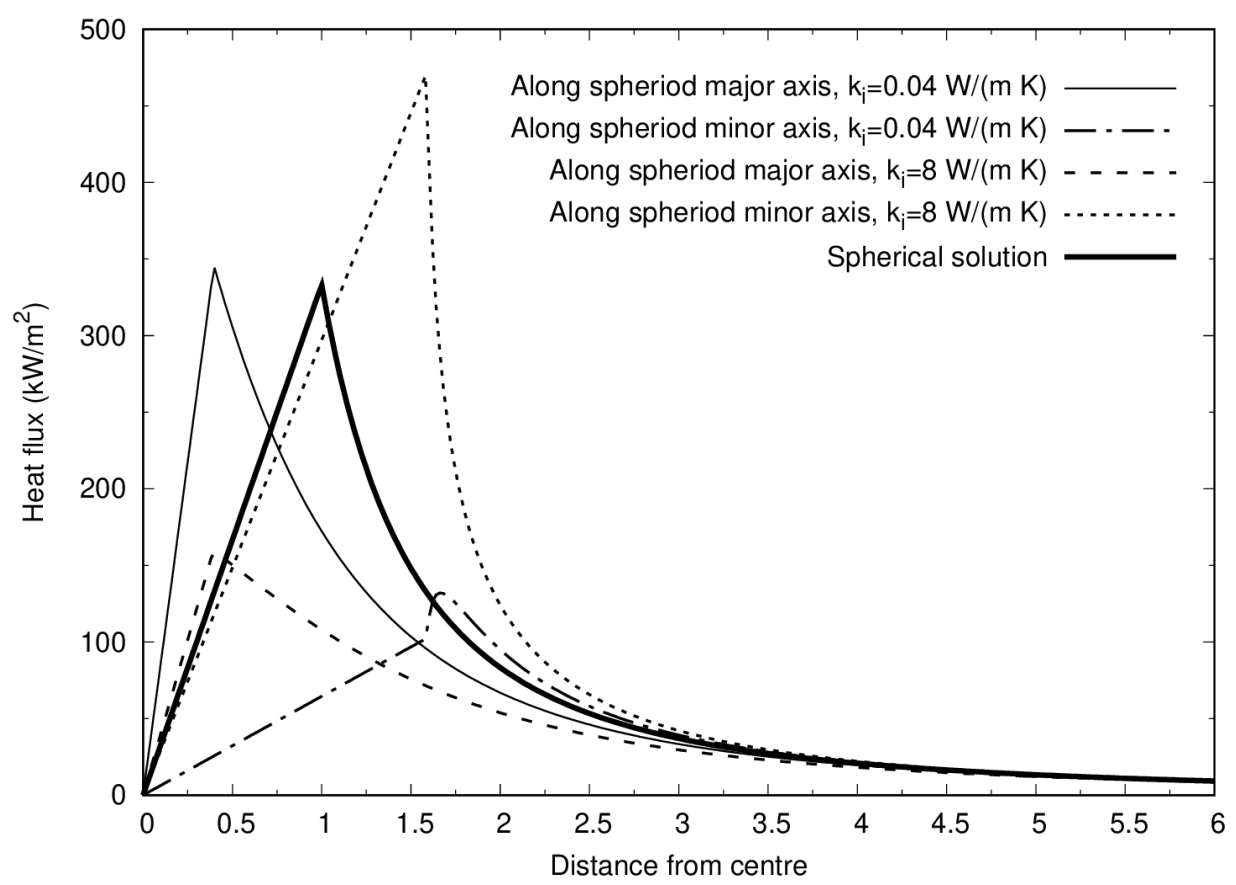

Figure 4: The heat flux magnitude variations along the major and minor axes of the prolate spheroids (a) $(D / d)=2$ and $(b)(D / d)=4$, and the oblate spheroids $(c)(D / d)=1 / 2$ and $(d)(D / d)=1 / 4$, and where the rest of the conditions are as in Fig. 2. 
(a)

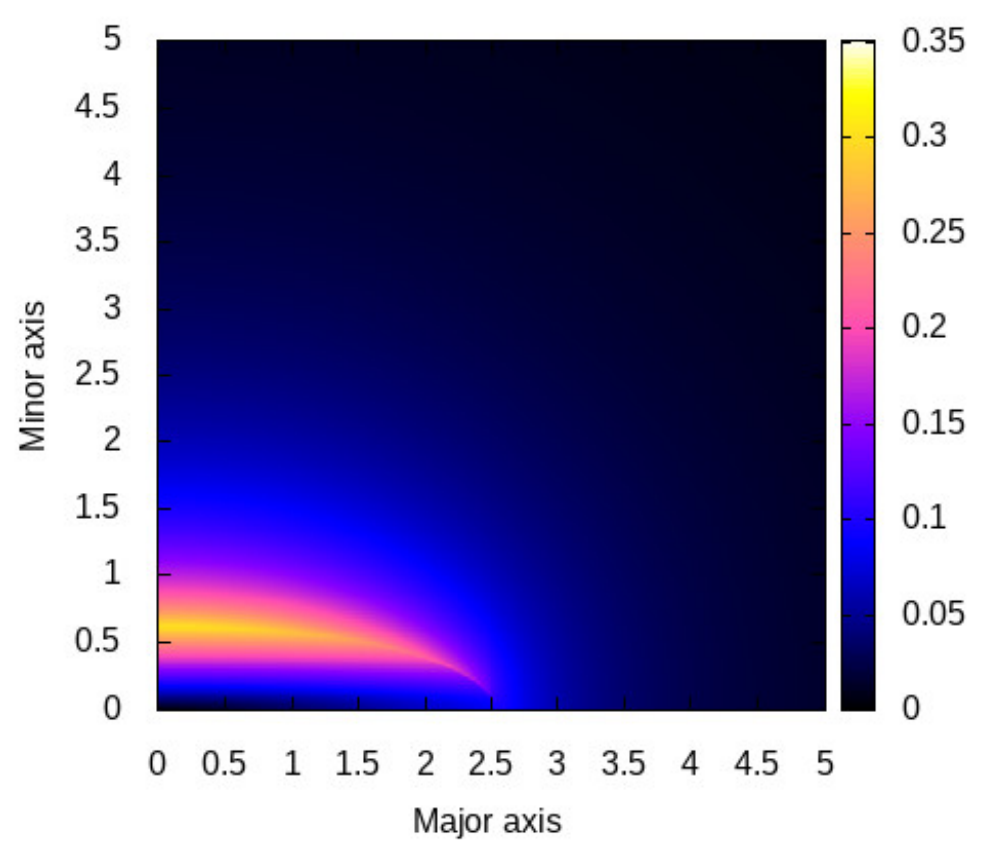

(b)

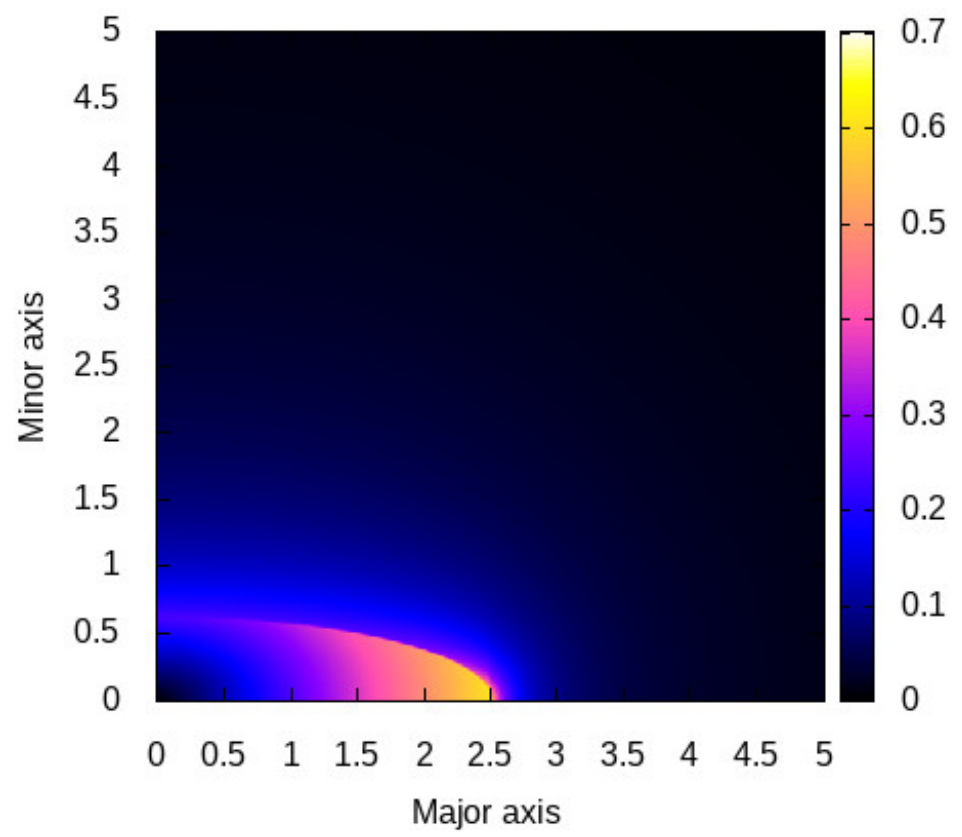

Figure 5: The heat flux contours in in $\mathrm{kW} / \mathrm{m}^{2}$ for the prolate spheroid of $\mathrm{D} / \mathrm{d}=4$ and where the spheroid's thermal conductivity is (a) $\mathrm{k}_{\mathrm{i}}=0.04 \mathrm{~W} /(\mathrm{m} \mathrm{K})$ and (b) $\mathrm{k}_{\mathrm{i}}=8 \mathrm{~W} /(\mathrm{m} \mathrm{K})$. The rest of the conditions are as in Fig. 2. 
(a)

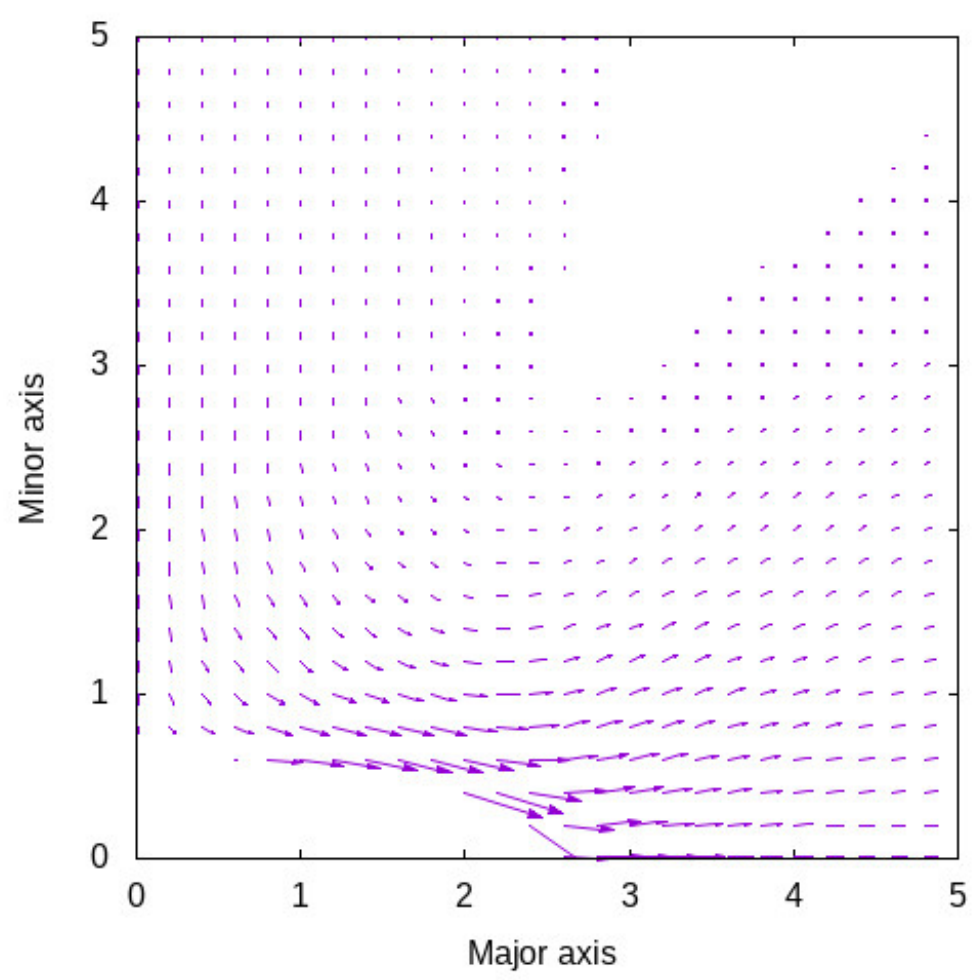

(b)

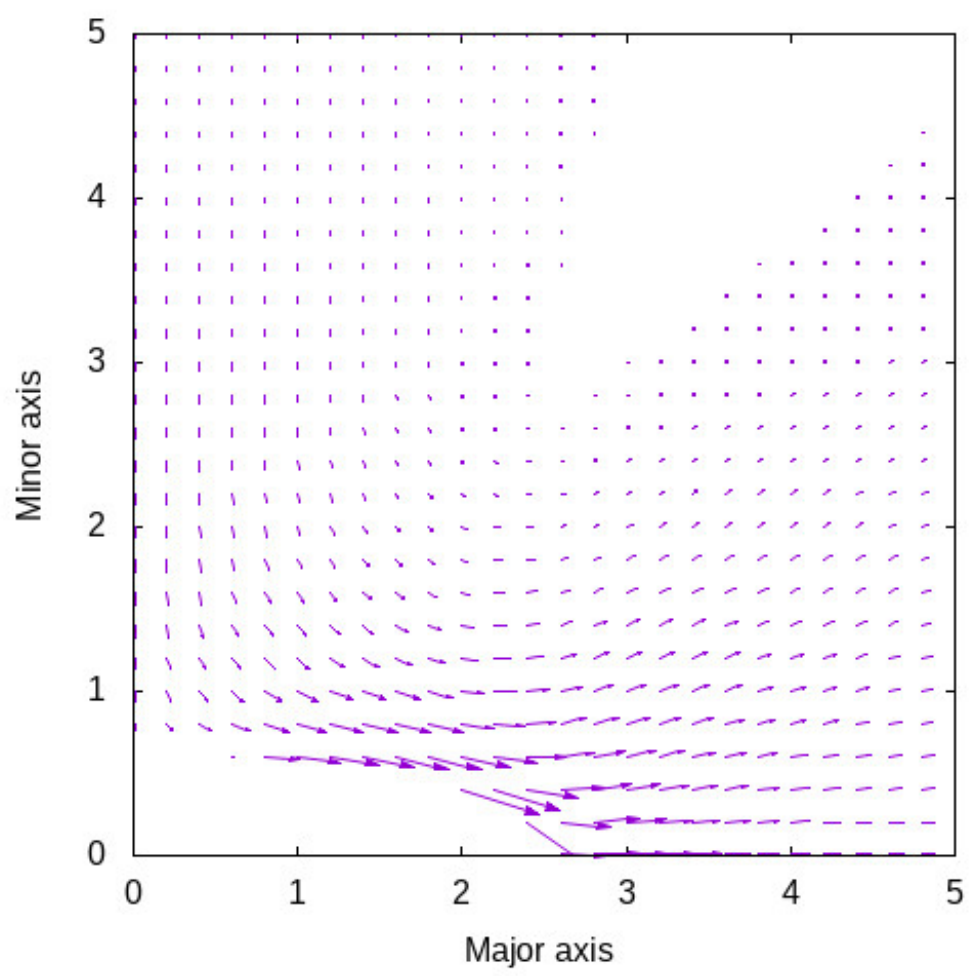

Figure 6: Induced velocity vectors that are plotted for the prolate spheroid of $D / d=4$ and where the spheroid's thermal conductivity is (a) $\mathrm{k}_{\mathrm{i}}=0.04 \mathrm{~W} /(\mathrm{m} \mathrm{K})$ and (b) $\mathrm{k}_{\mathrm{i}}=8 \mathrm{~W} /(\mathrm{m} \mathrm{K})$. The vector magnitude has been increased by $10 / 3$ for $k_{i}=8 \mathrm{~W} /(\mathrm{m} \mathrm{K})$ as compared to $\mathrm{k}_{\mathrm{i}}=0.04 \mathrm{~W} /(\mathrm{m} \mathrm{K})$. The rest of the conditions are as in Fig 2. 
(a)

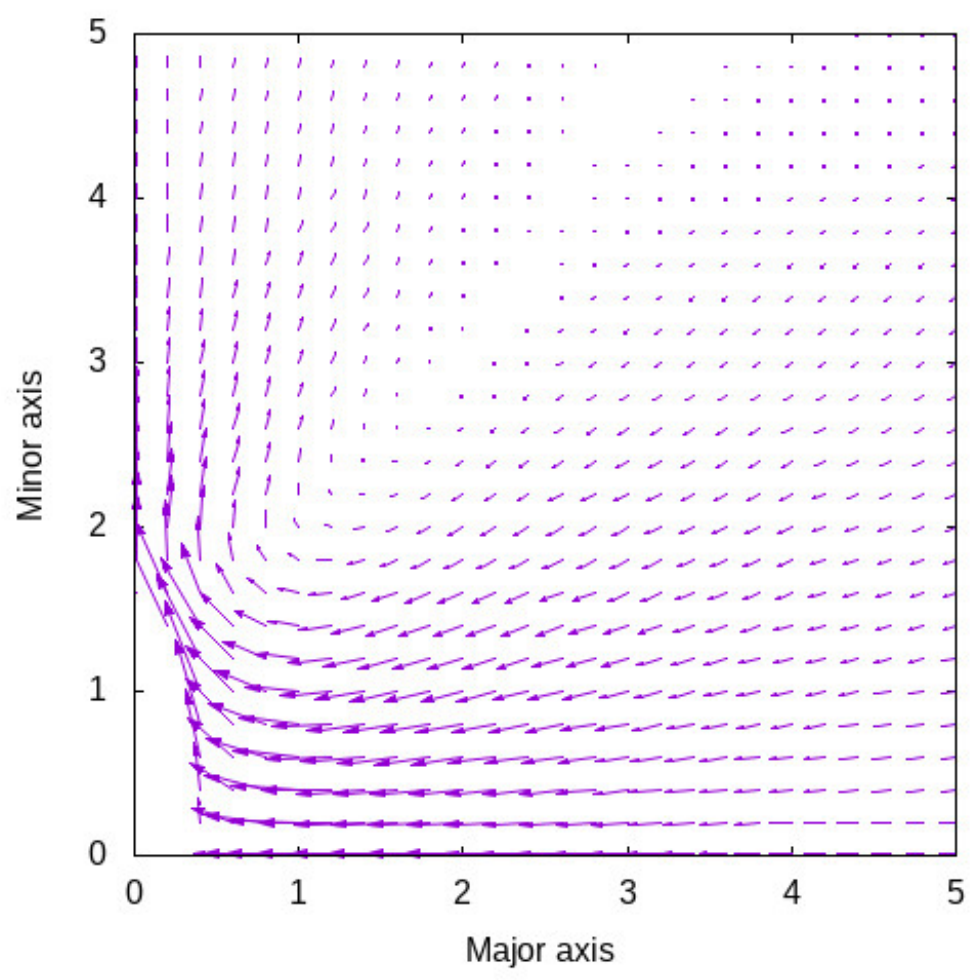

(b)

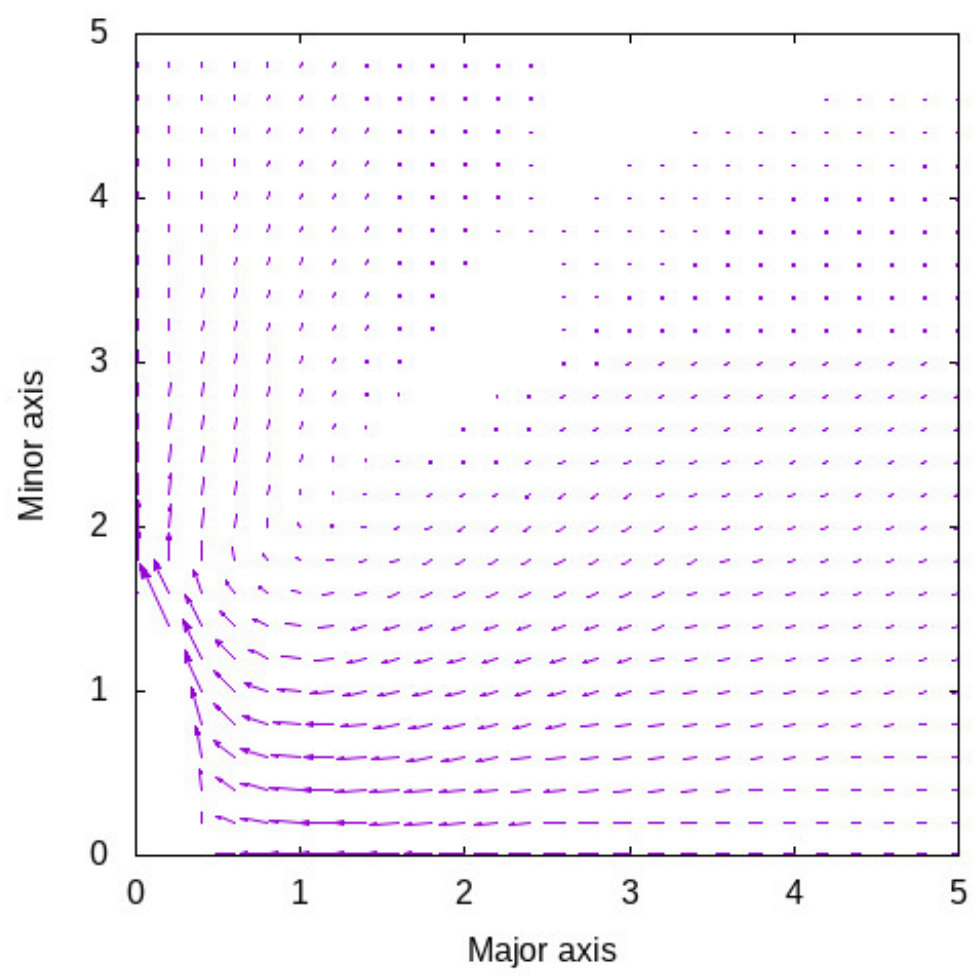

Figure 7: Induced velocity vectors that are plotted for the oblate spheroid of $D / d=1 / 4$ and where the spheroid's thermal conductivity is (a) $\mathrm{k}_{\mathrm{i}}=0.04 \mathrm{~W} /(\mathrm{m} \mathrm{K})$ and (b) $\mathrm{k}_{\mathrm{i}}=8 \mathrm{~W} /(\mathrm{m} \mathrm{K})$. The vector magnitude has been increased by $10 / 3$ for $k_{i}=8 \mathrm{~W} /(\mathrm{m} \mathrm{K})$ as compared to $\mathrm{k}_{\mathrm{i}}=0.04 \mathrm{~W} /(\mathrm{m} \mathrm{K})$. The rest of the conditions are as in Fig 2. 
(a)

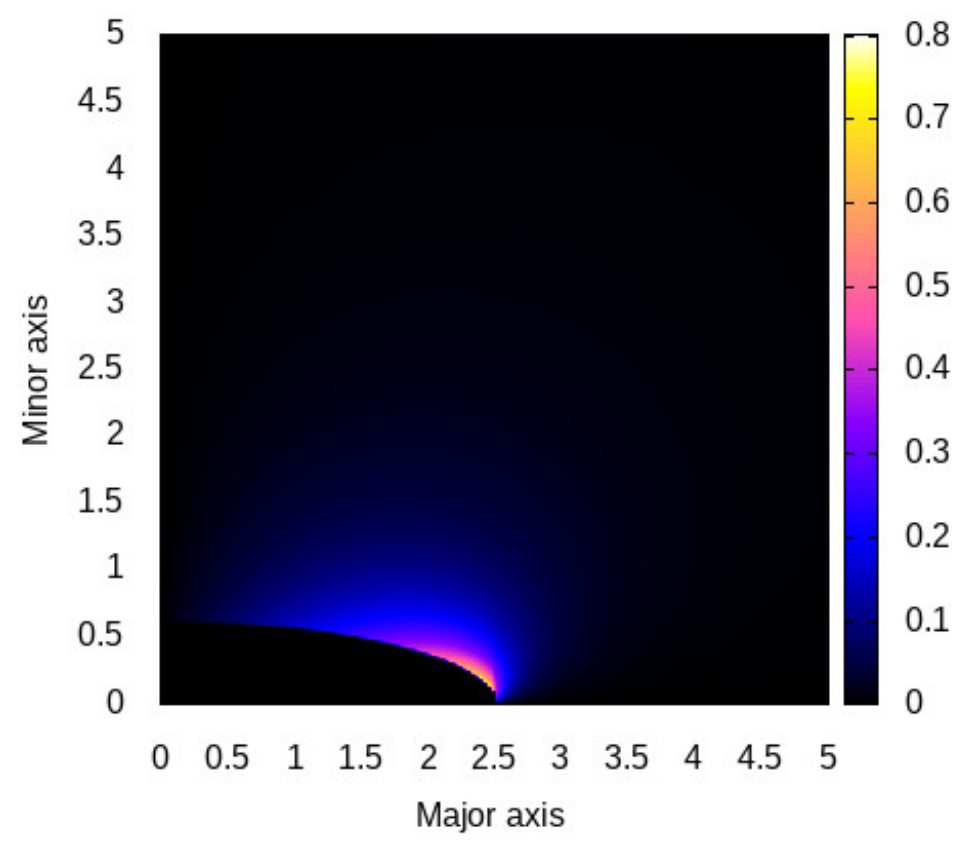

(b)

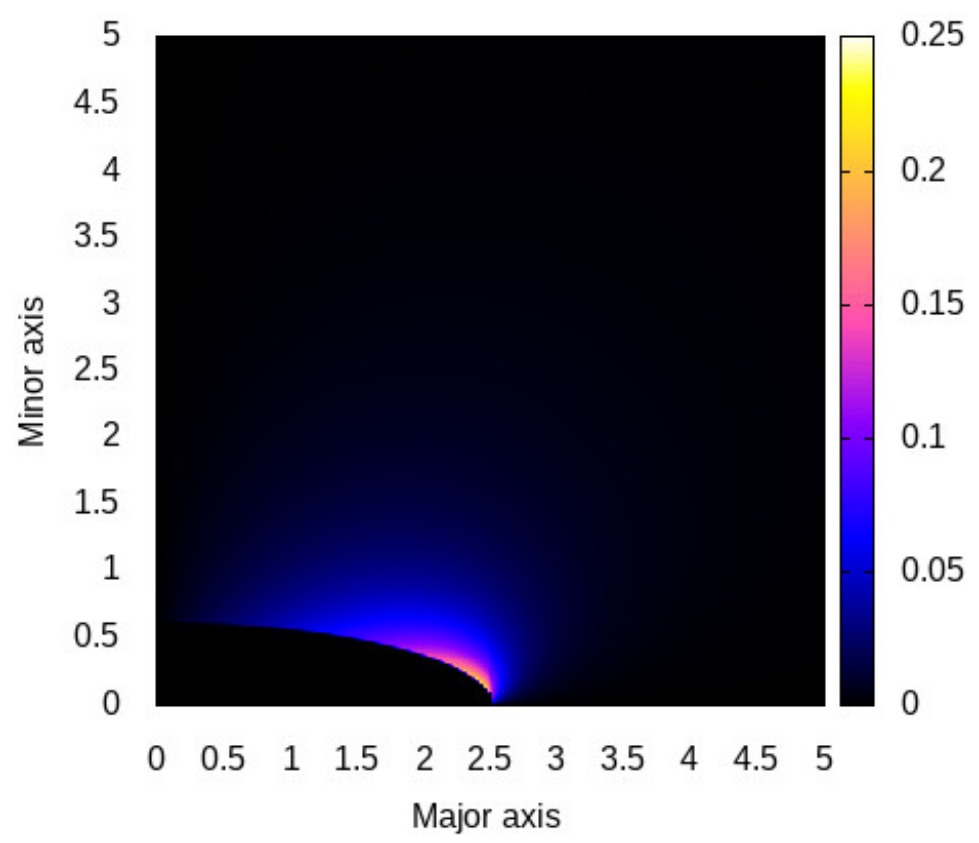

Figure 8: Contours of the vorticity in $1 / \mathrm{s}$ that are plotted for the prolate spheroids (a) $D / d=4$ and $\mathrm{k}_{\mathrm{i}}=0.04 \mathrm{~W} /(\mathrm{m} \mathrm{K})$, (b) $\mathrm{D} / \mathrm{d}=4$ and $\mathrm{k}_{\mathrm{i}}=8 \mathrm{~W} /(\mathrm{m} \mathrm{K})$. The rest of the conditions are as in Fig 2. 
(a)

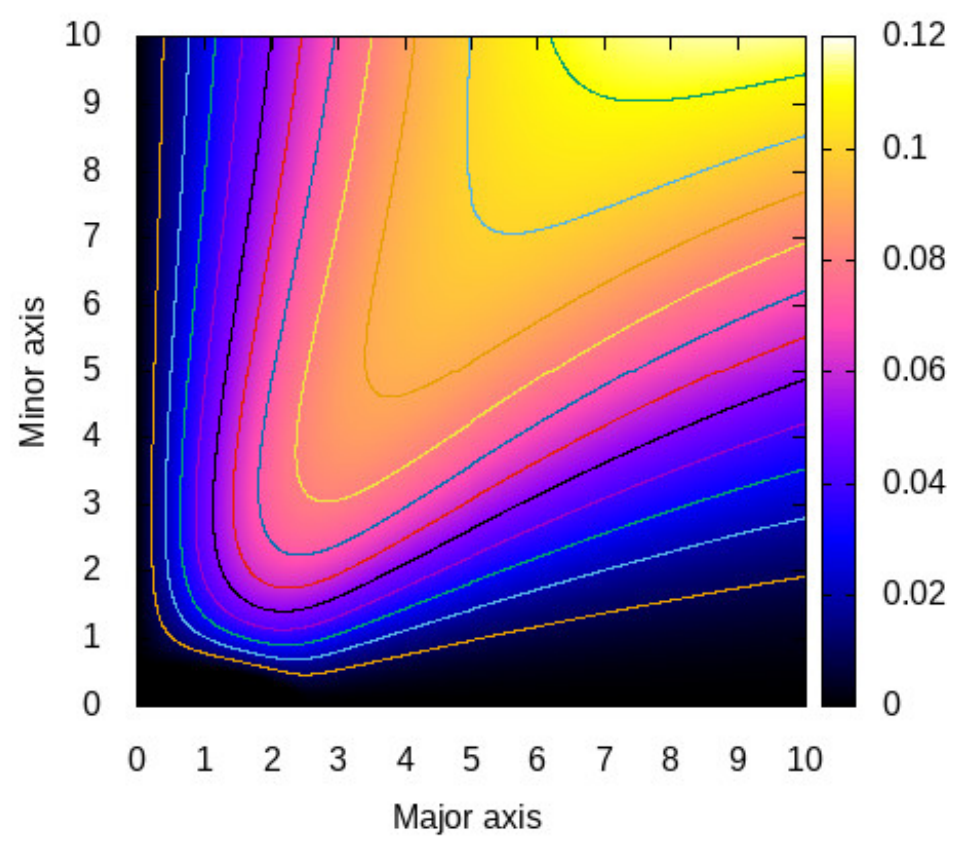

(b)

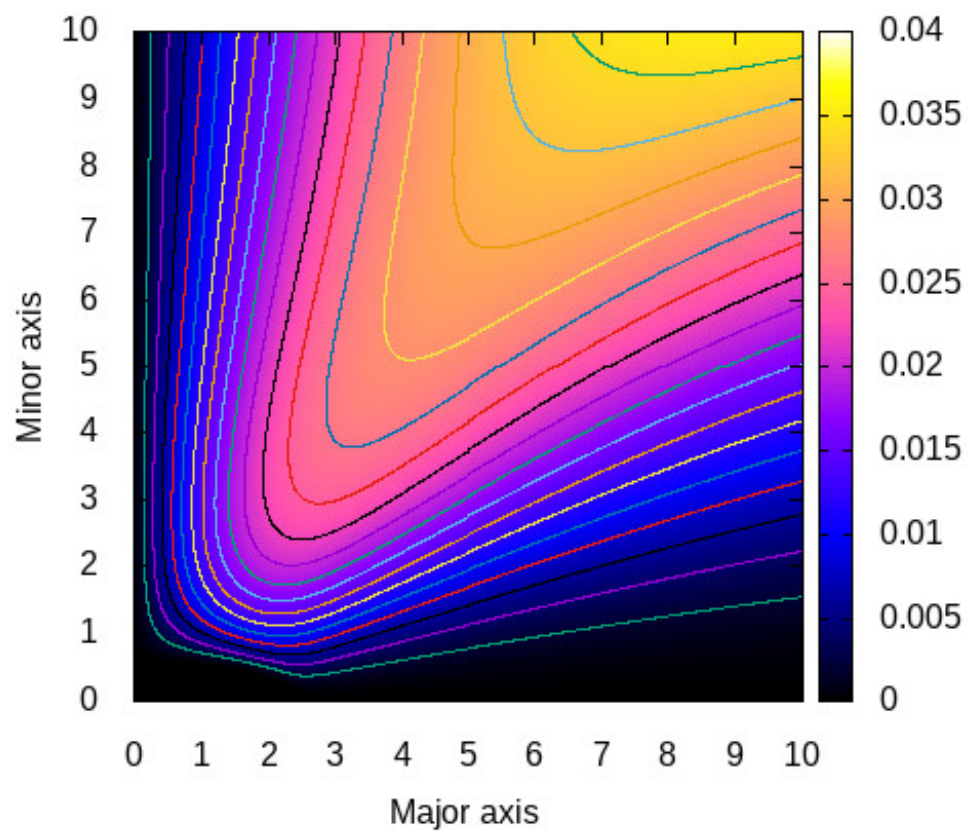

Figure 9: Contours of the stream-function in $\mathrm{m}^{2} / \mathrm{s}$ that are plotted for the prolate spheroids (a) $\mathrm{D} / \mathrm{d}=4$ and $\mathrm{k}_{\mathrm{i}}=0.04 \mathrm{~W} /(\mathrm{m} \mathrm{K})$, (b) $\mathrm{D} / \mathrm{d}=4$ and $\mathrm{k}_{\mathrm{i}}=8 \mathrm{~W} /(\mathrm{m} \mathrm{K})$. The rest of the conditions are as in Fig 2. 